\title{
Clumpy dust clouds and extended atmosphere of the AGB star W Hydrae revealed with VLT/SPHERE-ZIMPOL and VLTI/AMBER
}

\section{Time variations between pre-maximum and minimum light ${ }^{\star}$}

\author{
K. Ohnaka ${ }^{1}$, G. Weigelt ${ }^{2}$, and K.-H. Hofmann ${ }^{2}$ \\ 1 Universidad Católica del Norte, Instituto de Astronomía, 0610 Avenida Angamos, Antofagasta, Chile \\ e-mail: k1.ohnaka@gmail.com \\ 2 Max-Planck-Institut für Radioastronomie, Auf dem Hügel 69, 53121 Bonn, Germany \\ Received 20 September 2016 / Accepted 3 November 2016
}

\begin{abstract}
Aims. Our recent visible polarimetric images of the well-studied AGB star W Hya taken at pre-maximum light (phase 0.92) with VLT/SPHERE-ZIMPOL have revealed clumpy dust clouds close to the star at $\sim 2 R_{\star}$. We present second-epoch SPHERE-ZIMPOL observations of W Hya at minimum light (phase 0.54) as well as high-spectral resolution long-baseline interferometric observations with the AMBER instrument at the Very Large Telescope Interferometer (VLTI).

Methods. We observed W Hya with VLT/SPHERE-ZIMPOL at three wavelengths in the continuum $(645,748$, and $820 \mathrm{~nm})$, in the $\mathrm{H} \alpha$ line at $656.3 \mathrm{~nm}$, and in the TiO band at $717 \mathrm{~nm}$. The VLTI/AMBER observations were carried out in the wavelength region of the CO first overtone lines near $2.3 \mu \mathrm{m}$ with a spectral resolution of 12000 .

Results. The high-spatial resolution polarimetric images obtained with SPHERE-ZIMPOL have allowed us to detect clear time variations in the clumpy dust clouds as close as 34-50 mas (1.4-2.0 $\left.R_{\star}\right)$ to the star. We detected the formation of a new dust cloud as well as the disappearance of one of the dust clouds detected at the first epoch. The $\mathrm{H} \alpha$ and TiO emission extends to $\sim 150$ mas ( $\sim 6 R_{\star}$ ), and the $\mathrm{H} \alpha$ images obtained at two epochs reveal time variations. The degree of linear polarization measured at minimum light, which ranges from 13 to $18 \%$, is higher than that observed at pre-maximum light. The power-law-type limb-darkened disk fit to the AMBER data in the continuum results in a limb-darkened disk diameter of $49.1 \pm 1.5$ mas and a limb-darkening parameter of $1.16 \pm 0.49$, indicating that the atmosphere is more extended with weaker limb-darkening compared to pre-maximum light. Our Monte Carlo radiative transfer modeling shows that the second-epoch SPHERE-ZIMPOL data can be explained by a shell of $0.1 \mu \mathrm{m}$ grains of $\mathrm{Al}_{2} \mathrm{O}_{3}, \mathrm{Mg}_{2} \mathrm{SiO}_{4}$, and $\mathrm{MgSiO}_{3}$ with a $550 \mathrm{~nm}$ optical depth of $0.6 \pm 0.2$ and an inner and outer radii of $1.3 R_{\star}$ and $10 \pm 2 R_{\star}$, respectively. Our modeling suggests the predominance of small $(0.1 \mu \mathrm{m})$ grains at minimum light, in marked contrast to the predominance of large $(0.5 \mu \mathrm{m})$ grains at pre-maximum light.

Conclusions. The variability phase dependence of the characteristic grain size implies that small grains might just have started to form at minimum light in the wake of a shock, while the pre-maximum light phase might have corresponded to the phase of efficient grain growth.
\end{abstract}

Key words. techniques: polarimetric - techniques: interferometric - stars: imaging - stars: AGB and post-AGB circumstellar matter - stars: individual: W Hya

\section{Introduction}

Improving our understanding of dust formation is indispensable for solving the long-standing problem of the mass loss from asymptotic giant branch (AGB) stars. It is considered that the dust formation takes place within a few stellar radii, and observational studies of this region is crucial for clarifying the wind acceleration mechanism in AGB stars. The angular size of this innermost key region is smaller than $\sim 100$ mas even for nearby AGB stars, which means that we need high spatial resolution to study the dust formation zone.

The star W Hya is one of the closest AGB stars with a distance of $78_{-5.6}^{+6.5} \mathrm{pc}$ (Knapp et al. 2003), and therefore, it has been well studied from the visible to the radio. In the Simbad

* Based on SPHERE and AMBER observations made with the Very Large Telescope and Very Large Telescope Interferometer of the European Southern Observatory. Program ID: 095.D-0397(B) and 092.D-0461(A). database, W Hya is classified as a semi-regular variable. However, while its variability amplitude $(\Delta V \approx 3)$ is much smaller than that of typical Mira stars, clear periodicity can be seen in its light curve, and therefore, it is often classified as Mira in the literature. Recently we carried out polarimetric imaging of W Hya at five wavelengths from 645 to $820 \mathrm{~nm}$ with the VLT instrument SPHERE-ZIMPOL (Ohnaka et al. 2016, hereafter Paper I). Taking advantage of the superb performance of extreme adaptive optics (AO), we succeeded in spatially resolving clumpy dust clouds very close to the star at $\sim 50$ mas (just $\sim 2 R_{\star}$ ). Our $2 \mathrm{D}$ radiative transfer modeling suggests the predominance of large $(0.4-0.5 \mu \mathrm{m})$ grains of corundum $\left(\mathrm{Al}_{2} \mathrm{O}_{3}\right)$ or iron-poor silicate such as $\mathrm{Mg}_{2} \mathrm{SiO}_{4}$ and $\mathrm{MgSiO}_{3}$ and a density enhancement of a factor of $4 \pm 1$ in the clumps. Our results are consistent with the near-IR polarimetric interferometric observations obtained with the aperture-masking technique (Norris et al. 2012), which suggested the presence of grains of $0.3 \mu \mathrm{m}$ at $\sim 2 R_{\star}$. Grains such as $\mathrm{Al}_{2} \mathrm{O}_{3}, \mathrm{Mg}_{2} \mathrm{SiO}_{4}$, and $\mathrm{MgSiO}_{3}$ do not efficiently absorb stellar 
photons in the visible and in the near-IR due to their low opacities at these wavelengths. This means that the dust temperature can remain lower than the sublimation temperature, and therefore, the dust grains can form very close to the star. The detection of large, transparent grains close to the star lends support to the scenario proposed by Höfner (2008) that the radiation pressure due to the scattering of stellar photons-instead of absorption-by large, micron-sized grains can drive the mass loss in oxygen-rich AGB stars.

Dust formation in Mira-type AGB stars is strongly affected by the periodic passage of shocks generated by large-amplitude stellar pulsation, as demonstrated by self-consistent hydrodynamical models for AGB stellar winds (e.g., Fleischer et al. 1991, 1992, for carbon-rich stars, Jeong et al. 2003; and Höfner et al. 2016, for oxygen-rich stars). The nucleation and growth process of dust grains from the gas is a complicated, nonequilibrium process, which is not yet completely understood (e.g., Gobrecht et al. 2016; Gail et al. 2016). Therefore, studying time variations in the physical properties of the dust formation region is crucial for understanding the grain nucleation and growth process. The clear periodicity in the light curve of W Hya suggests the presence of pulsation, although its amplitude may be smaller than that of typical Miras. This means that we can expect the pulsation to cause shocks in the atmosphere of W Hya, which affect the formation of dust clouds.

In the present paper, we report on second-epoch polarimetric imaging observations of W Hya with VLT/SPHERE-ZIMPOL as well as high-spectral resolution long-baseline interferometric observations in the $\mathrm{CO}$ first overtone lines near $2.3 \mu \mathrm{m}$ with VLTI/AMBER to study time variations in the gas and dust environment in the immediate vicinity of the star. In Sect. 2, we describe the observations and data reduction. The observational results are presented in Sect. 3. The determination of the effective temperature and luminosity is described in Sect. 4. The radiative transfer modeling of the data is presented in Sect. 5, followed by interpretation of the results in Sect. 6 and concluding remarks in Sect. 7.

\section{Observations}

\subsection{Visible polarimetric imaging observations with SPHERE-ZIMPOL}

VLT/SPHERE allows us to carry out high-spatial resolution and high-contrast imaging, taking advantage of an extreme adaptive optics (AO) system for the wavelength range from 0.55 to $2.32 \mu \mathrm{m}$ (Beuzit et al. 2008). The ZIMPOL instrument is a unit for nearly diffraction-limited polarimetric imaging (also classical, non-polarimetric imaging) at 550-900 nm (Thalmann et al. 2008). Our SPHERE-ZIMPOL observations of W Hya (Program ID: 095.D-0397(B); P.I.: K. Ohnaka) occurred on 2016 March 23 (UTC). As in our first-epoch observations reported in Paper I, we used the P2 mode, in which the field orientation remains fixed. The $V$ magnitude of W Hya at the time of our second-epoch observations is estimated to be $\sim 9.5$, corresponding to phase 0.54 (minimum light), from the light curve of the American Association of Variable Star Observers (AAVSO). We observed the K1III star HIP $67834(V=7.6)$ as a reference of the point spread function (PSF). Given an angular diameter of $0.538 \pm 0.016$ mas $\left(\right.$ CalVin database $\left.{ }^{1}\right)$, this reference star should appear as a point source with the spatial resolution of SPHEREZIMPOL.

\footnotetext{
1 http://www . eso.org/observing/etc/bin/gen/form?INS. NAME $=$ CALVIN + INS . MODE $=$ CFP
}

As in our first-epoch observations, we used three filters to sample the (pseudo-)continuum regions relatively (but not entirely) free from the $\mathrm{TiO}$ bands ( $\mathrm{CntHa}$ with central wavelength $\lambda_{\mathrm{c}}=644.9 \mathrm{~nm}$ and $F W H M=4.1 \mathrm{~nm}$, Cnt748 with $\lambda_{\mathrm{c}}=747.4 \mathrm{~nm}$ and $F W H M=20.6 \mathrm{~nm}$, and Cnt820 with $\lambda_{\mathrm{c}}=817.3 \mathrm{~nm}$ and $F W H M=19.8 \mathrm{~nm})$. In addition, we observed with the NHa filter $\left(\lambda_{\mathrm{c}}=656.34 \mathrm{~nm}\right.$ and $\left.F W H M=0.97 \mathrm{~nm}\right)$ and the TiO717 filter $\left(\lambda_{\mathrm{c}}=716.8 \mathrm{~nm}\right.$ and $\left.F W H M=19.7 \mathrm{~nm}\right)$ to probe the $\mathrm{H} \alpha$ emission and TiO emission, respectively. The SPHERE-ZIMPOL instrument is equipped with two cameras (cam1 and cam2), with a pixel scale of 3.628 mas. This allows us to observe a given target with the same or different filters simultaneously. We observed W Hya by using the filter pairs of (CntHa, NHa), (TiO717, Cnt748), and (Cnt820, Cnt820). For each target and with each filter pair, we took $N_{\text {exp }}$ exposures for each of the Stokes $Q_{+}$, $Q_{-}, U_{+}$, and $U_{-}$components, with NDIT frames in each exposure. The polarization cycle of $Q_{+}, Q_{-}, U_{+}$, and $U_{-}$was repeated $N_{\text {pol }}$ times. We carried out this procedure $\left(N_{\exp } \times N_{\text {pol }}\right)$ at three different dithering positions.

The Strehl ratios of the observations of W Hya were estimated from the $H$-band Strehl ratios from the auxiliary files of SPHERE observations (so-called GEN-SPARTA data) as described in Paper I. The Strehl ratios of the observations of the PSF reference HIP 67834 were directly measured from the observed intensity maps. The Strehl ratios of the observations of W Hya are 0.38 (CntHa/NHa), 0.46 ( TiO717/Cnt748), and 0.52 (Cnt820/Cnt820), thanks to good seeing (0.7-0.8"). For the PSF reference, the values are lower, $0.25(\mathrm{CntHa} / \mathrm{NHa}), 0.36$ (TiO717/Cnt748), and 0.38 (Cnt820/Cnt820), because its flux at the wavelengths of the filters used in our observations is lower than that of W Hya. The summary of our SPHERE-ZIMPOL observations is given in Table 1.

We reduced the SPHERE-ZIMPOL data using the pipeline version $0.15 .0-2^{2}$ in the same manner as described in Paper I and obtained the maps of total (i.e., polarized + unpolarized) intensity $(I)$, polarized intensity $\left(I_{\mathrm{P}}\right)$, degree of linear polarization $\left(p_{\mathrm{L}}\right)$, and position angle of the polarization vector. In Paper I, we flux-calibrated the intensity maps using the visible spectrum of W Hya taken at a variability phase close to the SPHEREZIMPOL observations. However, we could not find a spectrum of W Hya covering the wavelengths of our SPHERE observations obtained near minimum light. Therefore, in the present work, we adopted the following approach. We first approximate the spectrum of the PSF reference star with a template spectrum with the corresponding effective temperature and the luminosity class available in the spectral library of Pickles (1998). Based on the effective temperature of $4548 \mathrm{~K}$ estimated for HIP 67834 (McDonald et al. 2012), we adopted the spectrum of a K2III star in the spectral library of Pickles (1998), whose effective temperature is the closest to HIP 67834 . We reddended the spectrum with $A_{V}=0.24$, which was obtained using the parameterization of the interstellar extinction of Arenou et al. (1992) and the wavelength-dependence of the interstellar extinction derived by Cardelli et al. (1989). Then the reddened template spectrum was scaled so that the flux computed with the $V$ band filter is equal to 7.64, which is the $V$ magnitude from Simbad. The resulting, flux-calibrated template spectrum allows us to compute the flux of our PSF reference star HIP 67834 with the SPHERE-ZIMPOL filters of our observations. Once the flux of HIP 67834 with the SPHERE-ZIMPOL filters is known, the images of W Hya can be flux-calibrated. The derived fluxes of W Hya are listed in

\footnotetext{
2 Available at ftp://ftp. eso.org/pub/dfs/pipelines/sphere
} 
K. Ohnaka et al.: Clumpy dust formation and molecular outer atmosphere of the AGB star W Hya. Time variations

Table 1. Summary of the SPHERE-ZIMPOL observations of W Hya.

\begin{tabular}{|c|c|c|c|c|c|c|c|c|c|c|c|}
\hline$\#$ & $\begin{array}{c}t_{\mathrm{obs}} \\
(\mathrm{UTC})\end{array}$ & $\begin{array}{l}\text { DIT } \\
(\mathrm{s})\end{array}$ & NDIT & $N_{\exp }$ & $N_{\mathrm{pol}}$ & $N_{\text {dither }}$ & $\begin{array}{l}\text { Filter } \\
(\text { cam } 1 / \text { cam } 2)\end{array}$ & $\begin{array}{l}\text { Seeing } \\
\left({ }^{\prime \prime}\right)\end{array}$ & $\mathrm{AM}$ & $\begin{array}{l}\text { Strehl } \\
(H)\end{array}$ & $\begin{array}{l}\text { Strehl } \\
\text { (visible) }\end{array}$ \\
\hline \multicolumn{12}{|c|}{ W Hya: 2016 March 23 (UTC) } \\
\hline 1 & $03: 58: 46$ & 20 & 6 & 1 & 1 & 3 & $\mathrm{CntHa} / \mathrm{NHa}$ & 0.73 & 1.16 & 0.86 & 0.38 \\
\hline 2 & 04:29:04 & 3 & 4 & 1 & 8 & 3 & $\mathrm{TiO} 717 / \mathrm{Cnt} 748$ & 0.74 & 1.09 & 0.86 & 0.46 \\
\hline 3 & $05: 05: 18$ & 1 & 12 & 1 & 6 & 3 & Cnt820/Cnt820 & 0.77 & 1.04 & 0.85 & 0.52 \\
\hline \multicolumn{12}{|c|}{ HIP 67834: 2016 March 23 (UTC) } \\
\hline $\mathrm{C} 1$ & $07: 10: 37$ & 10 & 10 & 1 & 1 & 3 & $\mathrm{CntHa} / \mathrm{NHa}$ & 0.63 & 1.02 & 0.74 & 0.25 \\
\hline $\mathrm{C} 2$ & 07:35:09 & 5 & 2 & 1 & 4 & 3 & TiO717/Cnt748 & 0.54 & 1.04 & 0.77 & 0.36 \\
\hline $\mathrm{C} 3$ & $07: 49: 30$ & 2 & 6 & 1 & 5 & 3 & Cnt820/Cnt820 & 0.69 & 1.07 & 0.73 & 0.38 \\
\hline
\end{tabular}

Notes. DIT: detector integration time. NDIT: number of frames. $N_{\text {exp }}$ : number of exposures of each polarization component in each polarization cycle at each dithering position. $N_{\text {pol }}$ : number of polarization cycles at each dithering position. $N_{\text {dither }}$ : number of dithering positions. Seeing was measured in the visible. AM: airmass. The Strehl ratios in the visible are computed from the $H$-band Strehl ratios for W Hya, while they were measured from the observed ZIMPOL images for HIP 67834.

Table 2. Flux of W Hya derived at the second epoch (phase 0.54, minimum light) with five filters of our SPHERE-ZIMPOL observations.

\begin{tabular}{llcc}
\hline \hline Filter & $\begin{array}{l}\lambda_{\mathrm{c}} \\
(\mathrm{nm})\end{array}$ & $\begin{array}{c}\text { Flux (phase 0.54) } \\
\left(\mathrm{W} \mathrm{m}^{-2} \mu \mathrm{m}^{-1}\right)\end{array}$ & $\begin{array}{c}\text { Flux (phase 0.92) } \\
\left(\mathrm{W} \mathrm{m}^{-2} \mu \mathrm{m}^{-1}\right)\end{array}$ \\
\hline $\mathrm{CntHa}$ & 644.9 & $5.62 \times 10^{-11}$ & $3.78 \times 10^{-10}$ \\
$\mathrm{NHa}$ & 656.34 & $6.40 \times 10^{-11}$ & $4.33 \times 10^{-10}$ \\
$\mathrm{TiO} 717$ & 716.8 & $2.22 \times 10^{-10}$ & $1.09 \times 10^{-9}$ \\
$\mathrm{Cnt748}$ & 747.4 & $5.30 \times 10^{-10}$ & $4.36 \times 10^{-9}$ \\
$\mathrm{Cnt820}$ & 817.3 & $5.29 \times 10^{-10}$ & $1.05 \times 10^{-8}$ \\
\hline
\end{tabular}

Notes. These values are used for the flux calibration of the SPHEREZIMPOL images. The flux obtained at the first epoch (phase 0.92, premaximum light) from Paper I is also given for reference.

Table 2, together with the fluxes obtained at the first epoch in Paper I.

\subsection{Long-baseline spectro-interferometric observations with VLTI/AMBER}

The near-IR (1.3-2.4 $\mu \mathrm{m})$ VLTI instrument AMBER (Petrov et al. 2007) allows us to achieve a spatial resolution of 3 mas (at $2 \mu \mathrm{m}$ ) with the current maximum baseline of $140 \mathrm{~m}$ by combining three Unit Telescopes (UTs) or $1.8 \mathrm{~m}$ Auxiliary Telescopes (ATs). The AMBER instrument operates with three spectral resolutions, 35, 1500, and 12000 . The highest spectral resolution of 12000 is sufficient to resolve individual atomic and molecular lines.

AMBER measures visibility, closure phase $(\mathrm{CP})$, and differential phase (DP). The visibility, which is the amplitude of the Fourier transform of the object's intensity distribution on the sky, contains information about the size and shape of the object. While the Fourier phase is destroyed by the atmospheric turbulence, the closure phase, which is the sum of the Fourier phases on three baselines around a triangle formed by three telescopes, conserves the phase information of the Fourier transform of the object's image. Deviations of CP from 0 or $180^{\circ}$ indicate asymmetry of the object. The DP represents the photocenter shift in spectral features with respect to the continuum. The AMBER instrument also records the spectrum of the same wavelength region simultaneously with the interferometric fringes.
Our VLTI/AMBER observations of W Hya took place on 2014 February 11 (UTC) with the AT configuration B2-C1-D0, which covered projected baseline lengths from 11.3 to $33.9 \mathrm{~m}$ (Program ID: 092.D-0461(A); P.I.: K. Ohnaka). Our AMBER observations covered the wavelength region between 2.28 and $2.31 \mu \mathrm{m}$ near the CO first overtone $2-0$ band head with the spectral resolution of 12000 . A summary of our AMBER observations is given in Table 3 . The variability phase at the time of our AMBER observations is estimated to be 0.59 from the AAVSO light curve (but in a different variability cycle, see Sect. 3.2 for discussion about the possible cycle-dependence of the angular size), which is close to the phase at the time of our second-epoch SPHERE-ZIMPOL observations. We observed $\alpha$ Cen A (G2V, $K=-1.5$, uniform-disk diameter $=8.314 \pm 0.016$ mas, Kervella et al. 2003) as an interferometric and spectroscopic calibrator.

The AMBER data were reduced using the amdlib ver $3.0 .7^{3}$, which is based on the P2VM algorithm (Tatulli et al. 2007; Chelli et al. 2009). Details of the reduction are described in Ohnaka et al. (2009, 2011, and 2013). The wavelength calibration and the spectroscopic calibration of the W Hya data were carried out with the method described in Ohnaka et al. (2013).

\section{Results}

\subsection{SPHERE-ZIMPOL polarimetric images}

\subsubsection{Clumpy dust clouds}

Figures 1-3 show the maps of total intensity $(I)$, polarized intensity $\left(I_{\mathrm{P}}\right)$, and degree of linear polarization $\left(p_{\mathrm{L}}\right)$ with the polarization vectors overlaid, respectively, obtained for W Hya. In each figure, we included the data from Paper I obtained at the first epoch (2015 July 8) corresponding to pre-maximum light (phase 0.92). In Fig. 1, the intensity maps of the PSF references are shown. The 2D Gaussian fit to the observed images of HIP 67834 results in PSF FWHMs of $22 \times 24$ mas at 645 and $656.3 \mathrm{~nm}, 23 \times 26 \mathrm{mas}$ at 717 and $748 \mathrm{~nm}$, and $26 \times 27$ mas at $820 \mathrm{~nm}$. We note that the Strehl ratios of the data of W Hya are similar for the observations at the first and second epochs: $\left(\mathrm{Strehl}_{2015}, \mathrm{Strehl}_{2016}\right)=(0.37,0.38)$ for the $\mathrm{CntHa} / \mathrm{NHa}$ pair, $(0.51,0.46)$ for the TiO717/Cnt748 pair, and $(0.39,0.52)$ for the Cnt820/Cnt820 pair. Therefore, our comparison of the images

3 http://www.jmmc.fr/data_processing_amber.htm 
Table 3. Summary of the VLTI/AMBER observations of W Hya and the calibrator $\alpha$ Cen A.

\begin{tabular}{ccccccc}
\hline \hline$\#$ & $\begin{array}{c}t_{\text {obs }} \\
(\mathrm{UTC})\end{array}$ & $\begin{array}{c}B_{\mathrm{p}} \\
(\mathrm{m})\end{array}$ & $\begin{array}{c}\text { PA } \\
\left({ }^{\circ}\right)\end{array}$ & $\begin{array}{c}\text { Seeing } \\
\left({ }^{\prime \prime}\right)\end{array}$ & $\begin{array}{c}\tau_{0} \\
(\mathrm{~ms})\end{array}$ & $\begin{array}{l}\text { DIT } \times N_{\mathrm{f}} \times N_{\text {exp }} \\
(\mathrm{ms})\end{array}$ \\
\hline \multicolumn{7}{c}{ W Hya: 2014 February $11(\mathrm{UTC})$} \\
1 & $08: 23: 25$ & $11.30 / 22.63 / 33.94$ & $21 / 21 / 21$ & 1.06 & 4.1 & $120 \times 500 \times 1$ \\
2 & $08: 25: 32$ & $11.30 / 22.64 / 33.94$ & $22 / 22 / 22$ & 1.06 & 4.1 & $120 \times 500 \times 1$ \\
3 & $08: 48: 33$ & $11.30 / 22.62 / 33.92$ & $24 / 24 / 24$ & 0.81 & 5.3 & $120 \times 500 \times 1$ \\
4 & $08: 50: 40$ & $11.30 / 22.62 / 33.92$ & $25 / 25 / 25$ & 0.94 & 4.6 & $120 \times 500 \times 1$ \\
5 & $08: 52: 48$ & $11.29 / 22.62 / 33.91$ & $25 / 25 / 25$ & 1.09 & 3.9 & $120 \times 500 \times 1$ \\
6 & $08: 54: 56$ & $11.29 / 22.61 / 33.91$ & $25 / 25 / 25$ & 0.98 & 4.4 & $120 \times 500 \times 1$ \\
7 & $08: 57: 02$ & $11.29 / 22.61 / 33.90$ & $25 / 25 / 25$ & 0.79 & 5.3 & $120 \times 500 \times 1$ \\
\hline \multicolumn{7}{c}{$\alpha$ Cen A: 2014 February $11(\mathrm{UTC})$} \\
C1 & $05: 02: 03$ & $10.07 / 20.17 / 30.23$ & $165 / 165 / 165$ & 1.02 & 4.8 & $120 \times 500 \times 5$ \\
C2 & $05: 36: 38$ & $10.14 / 20.32 / 30.46$ & $171 / 171 / 171$ & 0.97 & 5.0 & $120 \times 500 \times 5$ \\
C3 & $06: 51: 22$ & $10.19 / 20.40 / 30.59$ & $3 / 3 / 3$ & 0.94 & 4.9 & $120 \times 500 \times 5$ \\
C4 & $07: 26: 01$ & $10.15 / 20.33 / 30.48$ & $9 / 9 / 9$ & 0.82 & 5.5 & $120 \times 500 \times 5$ \\
C5 & $08: 02: 11$ & $10.07 / 20.18 / 30.25$ & $14 / 14 / 14$ & 1.26 & 3.5 & $120 \times 500 \times 5$ \\
C6 & $09: 06: 12$ & $9.83 / 19.69 / 29.52$ & $24 / 24 / 24$ & 0.98 & 4.2 & $120 \times 500 \times 5$ \\
C7 & $09: 40: 40$ & $9.64 / 19.31 / 28.95$ & $30 / 30 / 30$ & 0.84 & 3.8 & $120 \times 500 \times 5$ \\
\hline
\end{tabular}

Notes. $B_{\mathrm{p}}$ : projected baseline length. PA: position angle of the baseline vector projected onto the sky. DIT: detector Integration Time. $N_{\mathrm{f}}$ : number of frames in each exposure. $N_{\text {exp }}$ : number of exposures. The seeing and the coherence time $\left(\tau_{0}\right)$ were measured in the visible.

taken at two epochs is not severely affected by the difference in the AO performance.

The intensity maps of W Hya obtained at the second epoch (Fig. 1, third row) are significantly more extended than the PSF references, indicating that the circumstellar envelope is spatially resolved. While the peak of the intensity maps obtained at the first epoch is approximately at the center of the images (Fig. 1, top row), the peak of the images obtained at the second epoch is clearly off-centered. This off-centered peak can be a bright spot in the atmosphere of the central star or a bright dust clump. As described below, however, the polarized intensity maps, which reflects the spatial distribution of scattering material in optically thin cases, do not show a bright clump corresponding to the offcentered peak in the total intensity images. Therefore, it is more likely that it represents a bright spot in the atmosphere of the star. Similar inhomogeneities and their time variations within 48 days have recently been detected in another AGB star, R Dor, by Khouri et al. (2016). They interpret the inhomogeneities and their time variations as due to the fluctuations in the density and/or molecular abundance and/or excitation.

The polarized intensity maps reveal more detailed structures close to the star with the unpolarized light from the bright central star effectively suppressed. The $I_{P}$ maps shown in Fig. 2 reveal three clumpy dust clouds: a large, elongated bright clump at 43 mas away in the north, a smaller clump at 34 mas in the south, and an even smaller clump at 50 mas nearly in the west. The three clumps appear to be connected on the $I_{\mathrm{P}}$ map observed at $820 \mathrm{~nm}$. Given the star's angular diameter of $49.1 \pm 1.5$ mas measured with VLTI/AMBER (see Sect. 3.2), the location of the clumpy clouds correspond to $1.4-2.0 R_{\star}$, revealing again dust formation very close to the star. Comparison with the $I_{\mathrm{P}}$ maps obtained at the first epoch in 2015 reveals that while the overall appearance of the clumpy dust clouds is similar (i.e., a large clump in the north and a smaller clump in the south), there are variations in the detailed structure. On the one hand, the small clump detected in the west of the star at the second epoch is not seen in the data obtained at the first epoch, and therefore, it must have formed in the last 8.5 months. On the other hand, the clump seen in the SW of the star in 2015 has nearly disappeared. The relative errors in the polarized intensity on the bright clumps are $4-5 \%, 7-10 \%, 6-9 \%, 5-8 \%$, and $4-6 \%$ at $645,656.3,717,748$, and $820 \mathrm{~nm}$, respectively.

The FWHMs measured at 645, 656.3, 717, 748, and $820 \mathrm{~nm}$ at minimum light in 2016 are 68, 68, 72, 70, and 64 mas, respectively. These values are noticeably larger than the FWHMs measured in 2015 at pre-maximum: 53 mas at $645 \mathrm{~nm}, 51$ mas at $656.3 \mathrm{~nm}, 58$ mas at $717 \mathrm{~nm}$, and 46 mas at $820 \mathrm{~nm}$ (the central region of the 2015 image at $748 \mathrm{~nm}$ was affected by saturation). Therefore, our two-epoch observations suggest that the object (including the star itself, extended atmosphere, and dust clouds) appeared more extended at minimum light than at premaximum light. Moreover, the above FWHMs measured at minimum light are significantly larger than the values obtained by Ireland et al. (2004) - 75 mas (717 nm), 50 mas (748 nm), and 42 mas $(820 \mathrm{~nm})$ - except for $717 \mathrm{~nm}$, although they observed near minimum light (phase 0.44). Given that the size measured in the visible is significantly affected by the dust clouds, the difference between the FWHMs measured in our present work and Ireland et al. (2004) implies significant cycle-dependence of dust formation.

The $p_{\mathrm{L}}$ maps shown in Fig. 3 are characterized by an incomplete, asymmetric ring, which suggests an approximately shelllike distribution of dust. This is supported by the concentric pattern of the polarization vector maps. The maximum degree of linear polarization measured in 2016 is $18 \%, 16 \%, 13 \%, 14 \%$, and $16 \%$ at $645,656.3,717,748$, and $820 \mathrm{~nm}$, respectively. The absolute errors in the degree of linear polarization are $0.8 \%$ (i.e., $18 \% \pm 0.8 \%), 1 \%, 1 \%, 0.7 \%$, and $0.6 \%$ at $645,656.3,717,748$, and $820 \mathrm{~nm}$, respectively, in the region within $\sim 180$ mas. In the region beyond this, the absolute errors are higher, $2.5 \%, 5 \%$, $3 \%, 2 \%$, and $1.3 \%$ at $645,656.3,717,748$, and $820 \mathrm{~nm}$, respectively. The maximum degree of polarization measured in 2016 are systematically higher than those measured in 2015: 13\%, $12 \%, 10 \%, 9 \%$, and $8 \%$ at $645,656.3,717,748$, and $820 \mathrm{~nm}$, 
K. Ohnaka et al.: Clumpy dust formation and molecular outer atmosphere of the AGB star W Hya. Time variations
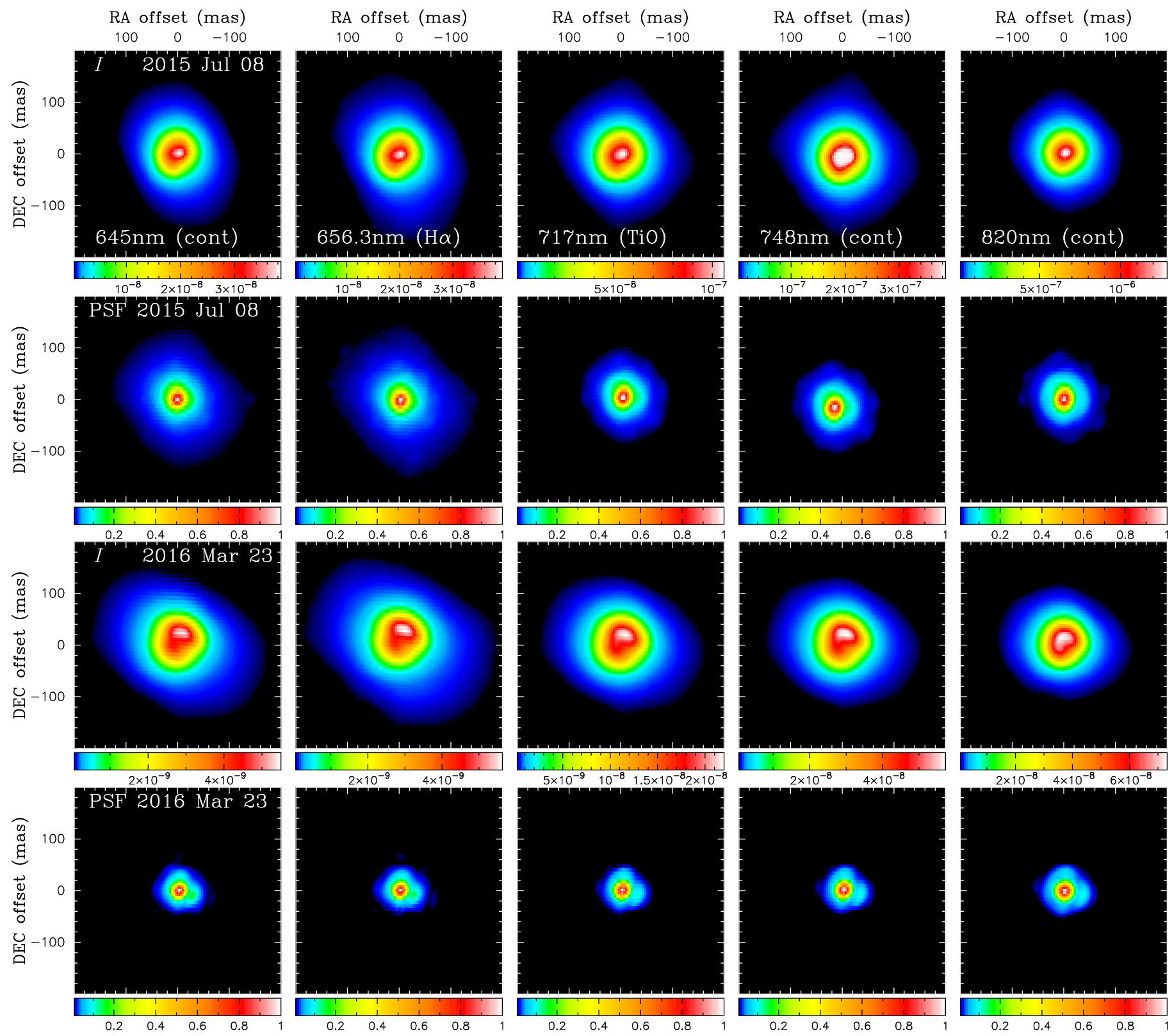

Fig. 1. Intensity maps of W Hya observed with SPHERE-ZIMPOL. The first and third rows show the data of W Hya obtained on 2015 July 8 (phase 0.92, Paper I) and on 2016 March 23 (phase 0.54), respectively. The second and fourth rows show the intensity maps of the PSF reference star observed in 2015 and 2016, respectively. The observed images at $645 \mathrm{~nm}$ (CntHa, continuum), $656.3 \mathrm{~nm}(\mathrm{NHa}, \mathrm{H} \alpha), 717 \mathrm{~nm}$ (TiO717, TiO band), $748 \mathrm{~nm}$ (Cnt748, continuum), and $820 \mathrm{~nm}$ (Cnt820, continuum) are shown from the left to the right. North is up, east to the left in all panels. While the intensity peak is approximately at the center in 2015, the peak is clearly off-centered in 2016. The Strehl ratios for W Hya are comparable between 2015 and 2016, although those for the PSF references differ significantly between 2015 and 2016 (Sect. 3.1 .1 ). The intensity maps of W Hya, which are flux-calibrated as described in Sect. 2.1, are shown in units of $\mathrm{W} \mathrm{m}^{-2} \mu \mathrm{m}^{-1} \operatorname{arcsec}^{-2}$. The color scale of the intensity maps is cut off at $1 \%$ of the intensity peak.

respectively. Furthermore, as can be seen in Fig. 4, while the polarization was detected only up to $\sim 150$ mas from the central star in 2015 , it extends to $\sim 250$ mas in 2016. This indicates that the distribution of the scattering material is more extended at phase 0.54 in 2016 than at phase 0.92 in 2015.

This can be interpreted as the consequence of dust formation in a more extended region in 2016 than in 2015 or the result of the outward motions of the dust clouds detected in 2015 However, the angular displacement expected over the time interval of 8.5 months between our two-epoch observations from the expansion velocity $v_{\exp }\left(\mathrm{km} \mathrm{s}^{-1}\right)$ and the distance of $78 \mathrm{pc}$ is $1.9 \times v_{\exp }$ mas. Since $v_{\exp }$ is expected to be smaller than the terminal velocity of $7.5 \mathrm{~km} \mathrm{~s}^{-1}$ (Khouri et al. 2015), the angular displacement is smaller than 14 mas. This means that we cannot explain the detection of polarization in a more extended region in 2016 by means of the expansion of the dust clouds detected in 2015. Therefore, it is more likely that the dust formation had taken place at larger radii at the time of our second-epoch observations compared to the first-epoch observations.

\subsubsection{Extended $\mathrm{H} \alpha$ and $\mathrm{TiO}$ emission}

The simultaneous observations of the filter pairs (CntHa, $\mathrm{NHa}$ ) and (TiO717, Cnt748) allow us to subtract the continuum image 

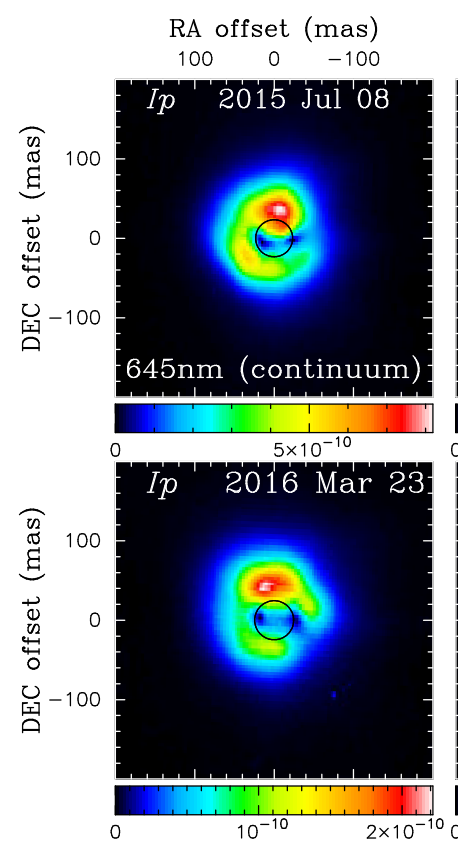

RA offset (mas)
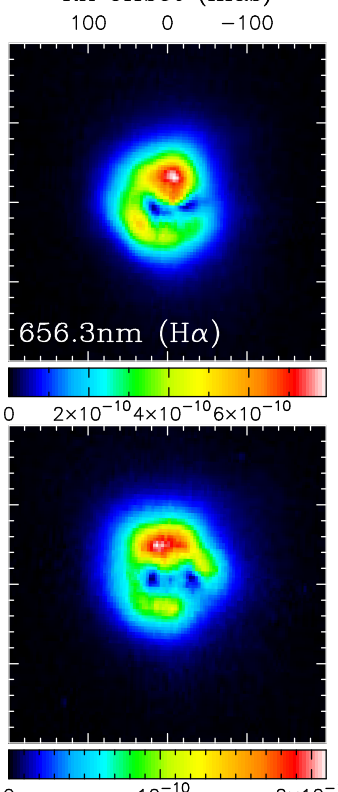

RA offset (mas)
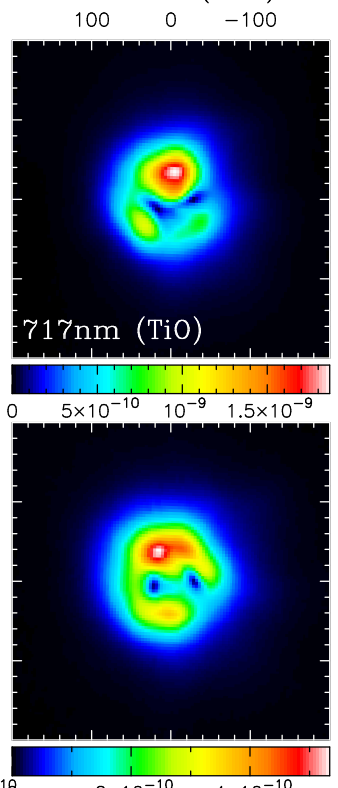

RA offset (mas)

$\begin{array}{lll}100 \quad 0 & -100\end{array}$
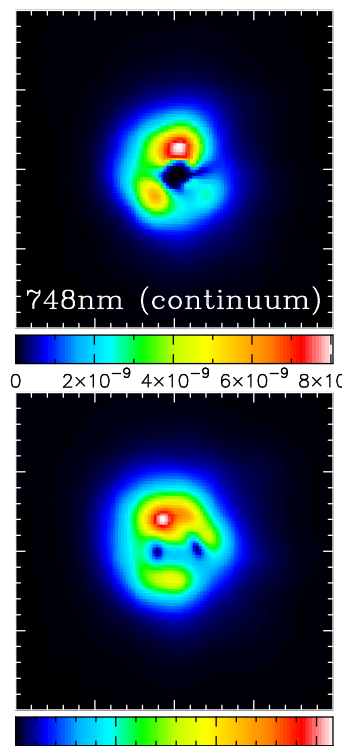

RA offset (mas)
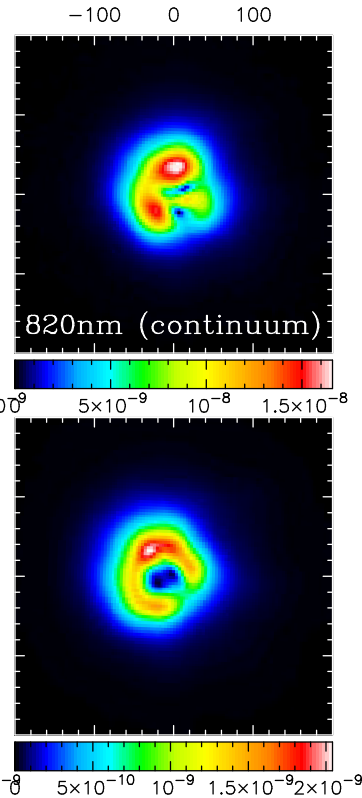

Fig. 2. Polarized intensity maps of W Hya observed with SPHERE-ZIMPOL. The top and bottom rows show the data of W Hya obtained on 2015 July 8 (phase 0.92, Paper I) and on 2016 March 23 (phase 0.54), respectively. The observed images at $645 \mathrm{~nm}$ (CntHa, continuum), $656.3 \mathrm{~nm}$ ( $\mathrm{NHa}, \mathrm{H} \alpha), 717 \mathrm{~nm}$ (TiO717, TiO band), $748 \mathrm{~nm}$ (Cnt748, continuum), and $820 \mathrm{~nm}$ (Cnt820, continuum) are shown from the left to the right. North is up, east to the left in all panels. The 2016 data reveal the formation of a new, small dust clump in the west, while the dust clump detected in the SW in 2015 disappeared in 2016. The polarized intensity is shown in units of $\mathrm{W} \mathrm{m}^{-2} \mu \mathrm{m}^{-1} \mathrm{arcsec}^{-2}$. The circles in the leftmost panels represent the size of the star measured in the continuum near $2.3 \mu \mathrm{m}$ based on our VLTI/AMBER observations.
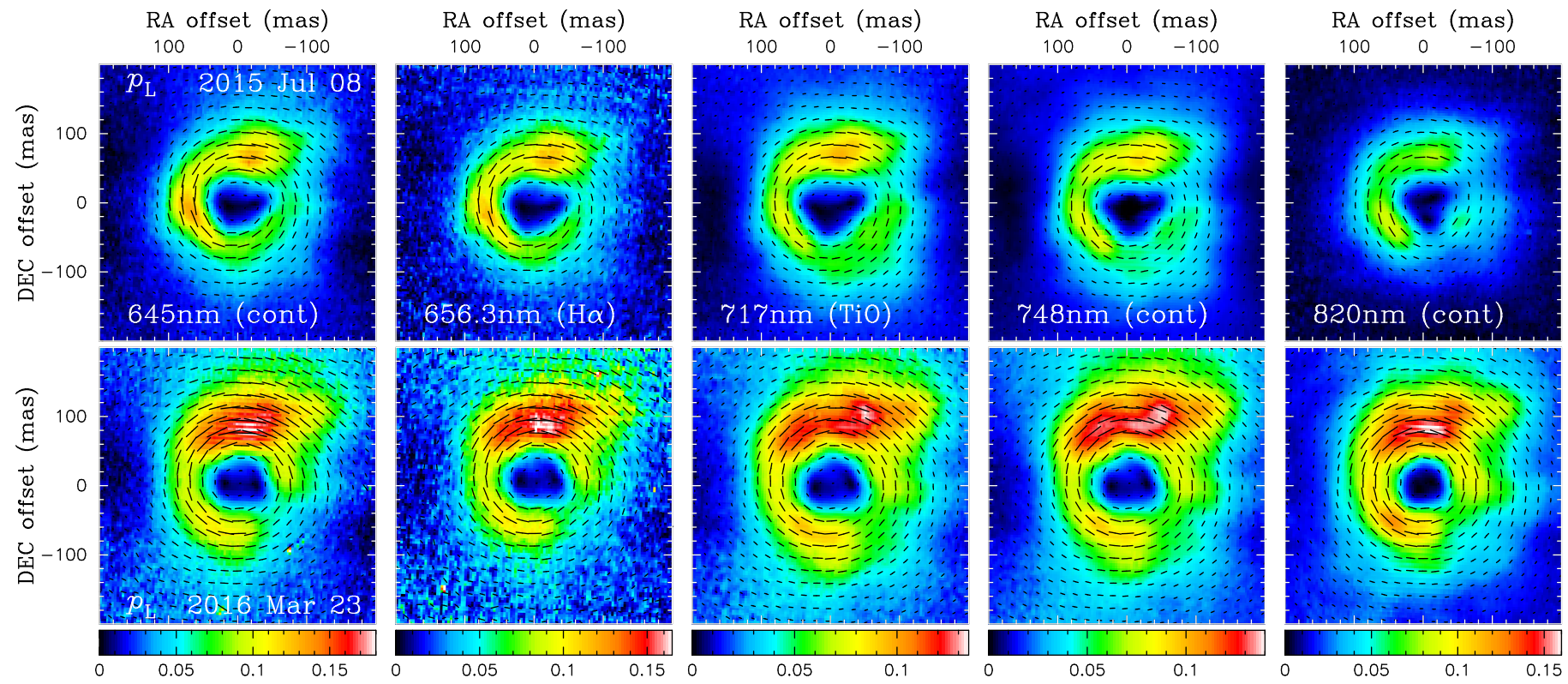

Fig. 3. Degree of linear polarization maps of W Hya observed with SPHERE-ZIMPOL. The top and bottom rows show the data of W Hya obtained on 2015 July 8 (phase 0.92, Paper I) and on 2016 March 23 (phase 0.54), respectively. The images observed at $645 \mathrm{~nm}$ (CntHa, continuum), $656.3 \mathrm{~nm}(\mathrm{NHa}, \mathrm{H} \alpha), 717 \mathrm{~nm}$ (TiO717, TiO band), $748 \mathrm{~nm}$ (Cnt748, continuum), and $820 \mathrm{~nm}$ (Cnt820, continuum) are shown from the left to the right. At each wavelength, the same color scale is used for the 2015 and 2016 data, which makes it easier to recognize that the degree of polarization measured in 2016 is systematically higher than that measured in 2015 . North is up, east to the left in all panels.

to study the extended $\mathrm{H} \alpha$ and $\mathrm{TiO}$ emission in detail, because the AO performance was the same for each pair. We subtracted the continuum image taken with the CntHa filter from the $\mathrm{NHa}$ image, in which both images are flux-calibrated as described in Sect. 2.1. Comparison of Figs. 5a and b reveals that the images taken at two epochs show strong, nearly circular $\mathrm{H} \alpha$ emission within $\sim 50$ mas $\left(\sim 2 R_{\star}\right)$. We detected fainter, more irregular-shaped emission extending to $\sim 200$ mas $\left(\sim 8 R_{\star}\right)$ and $\sim 150$ mas $\left(\sim 6 R_{\star}\right)$ at pre-maximum light in 2015 and at minimum light in 2016, respectively. The image taken in 2016 shows more emission in the E-W direction compared to the data taken in 2015 .

While the overall spatial extent of the $\mathrm{H} \alpha$ emission is comparable at both epochs, there are time variations in the 
K. Ohnaka et al.: Clumpy dust formation and molecular outer atmosphere of the AGB star W Hya. Time variations
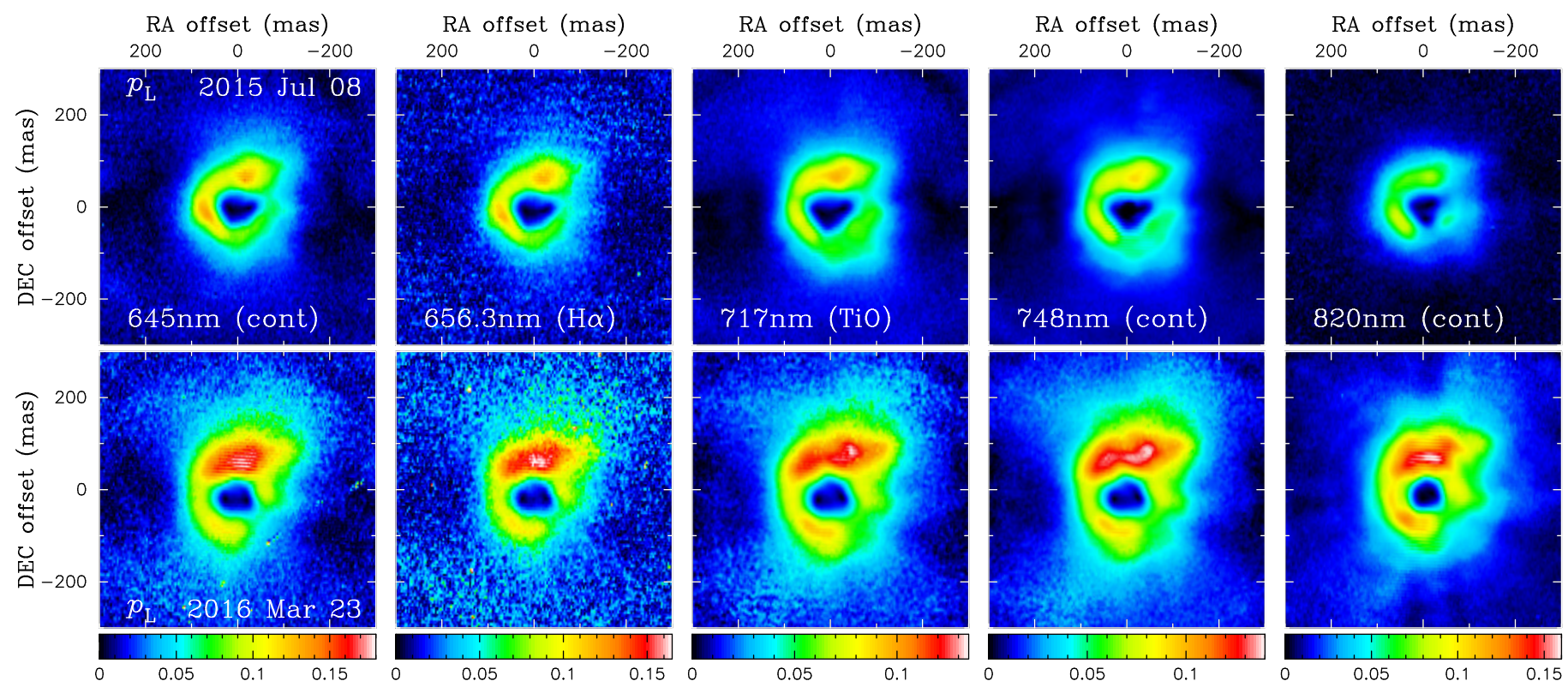

Fig. 4. Degree of linear polarization maps of W Hya. Same as Fig. 3 but with a larger field of view to show the entire extent of the polarization signals, which is noticeably more extended in 2016 than in 2015.

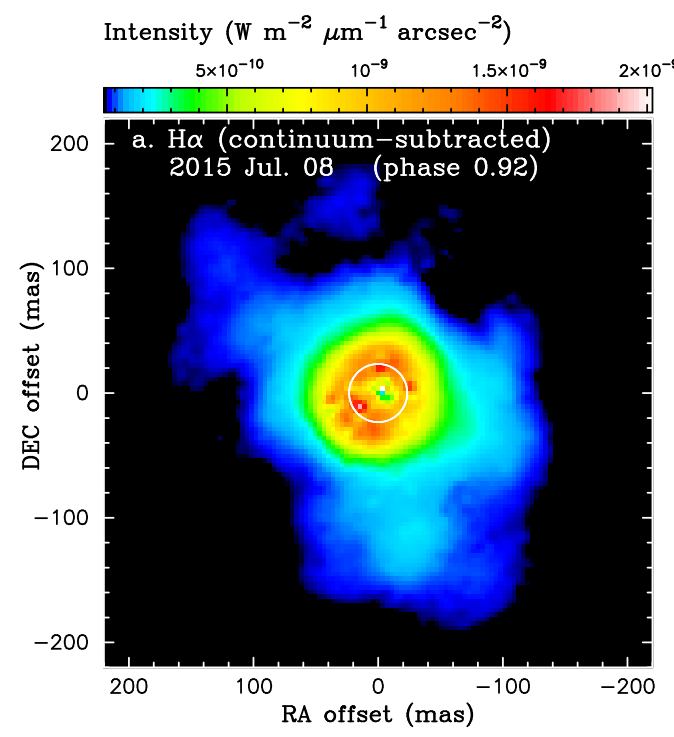

Intensity ( $\mathrm{W} \mathrm{m}^{-2} \mu \mathrm{m}^{-1} \operatorname{arcsec}^{-2}$ )

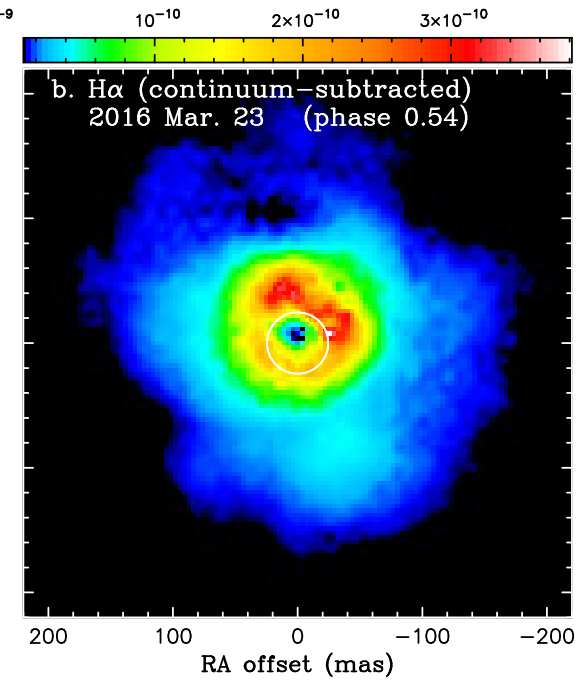

Normalized intensity

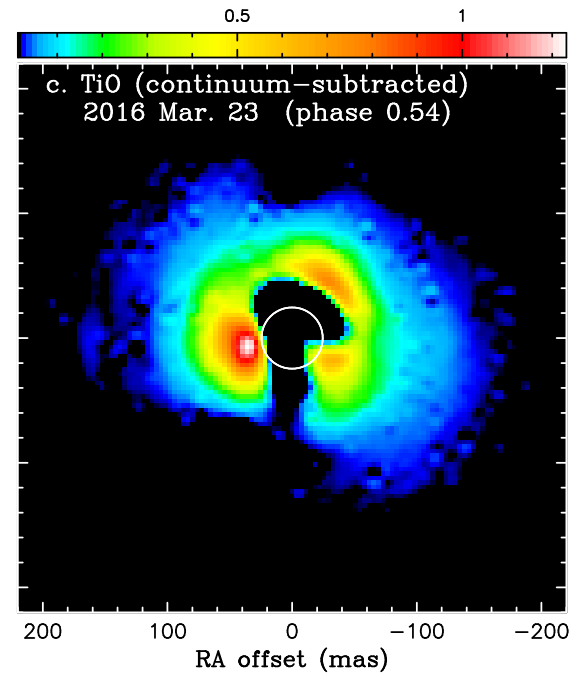

Fig. 5. a) Continuum-subtracted $\mathrm{H} \alpha$ image of W Hya derived from the SPHERE-ZIMPOL observations at pre-maximum light (phase 0.92) on 2015 July 08 (Paper I) . b) Continuum-subtracted H $\alpha$ image of W Hya observed at minimum light (phase 0.54) on 2016 March 23. c) Continuumsubtracted $\mathrm{TiO}(717 \mathrm{~nm}$ ) image of W Hya observed at minimum light (phase 0.54) on 2016 March 23. North is up, east to the left.

clumpy structures. For example, the clumpy emission detected at $\sim 125$ mas $\left(\sim 5.4 R_{\star}\right)$ in the SSW in 2015 appears at an angular distance of $\sim 115$ mas $\left(\sim 4.7 R_{\star}\right)$ at approximately the same position angle in 2016. The $\mathrm{H} \alpha$ emission is considered to be associated with shocks induced by stellar pulsation (e.g., Fox \& Wood 1984). In Paper I, we interpreted the extended H $\alpha$ emission as originating from the shocks propagating to as far as $\sim 5 R_{\star}$. However, as the shocks are significantly weakened at such large radii, it is more likely that the $\mathrm{H} \alpha$ emission originates from the shocks much closer to the star and is scattered at large radii probably by dust. Possibly the strong, nearly circular emission within $\sim 50$ mas $\left(\sim 2 R_{\star}\right)$ may represent the direct emission from the shocks. In this scenario, the $\mathrm{H} \alpha$ emission level is expected to change with pulsation phase, which is indeed observed, while the dependence on pulsation phase should be smeared out and be weak if the emission originates from the shocks far away from the star.

The $\mathrm{H} \alpha$ emission seems to be more extended at the position angles of the gaps between the dust clouds seen in the $I_{\mathrm{P}}$ maps (Fig. 2). The $\mathrm{H} \alpha$ emission in 2016 shows more extension to the east, SSW, and west, which approximately - though not exactly - corresponds to the gaps between the dust clouds. Similar anticorrelation is seen in the 2015 data as well. The extended $\mathrm{H} \alpha$ emission toward the south, SWW, and NE seems to correspond to the cloud gaps, which is particularly clear in the $I_{\mathrm{P}}$ map at $717 \mathrm{~nm}$ (Fig. 2, upper row, third column). If the extended $\mathrm{H} \alpha$ emission beyond $\sim 50$ mas represents the $\mathrm{H} \alpha$ photons that originate in the inner shocks and are scattered by dust grains at large radii, we can expect that the inner dust clouds can partially block the $\mathrm{H} \alpha$ photons, leading to weaker emission at large radii in the 

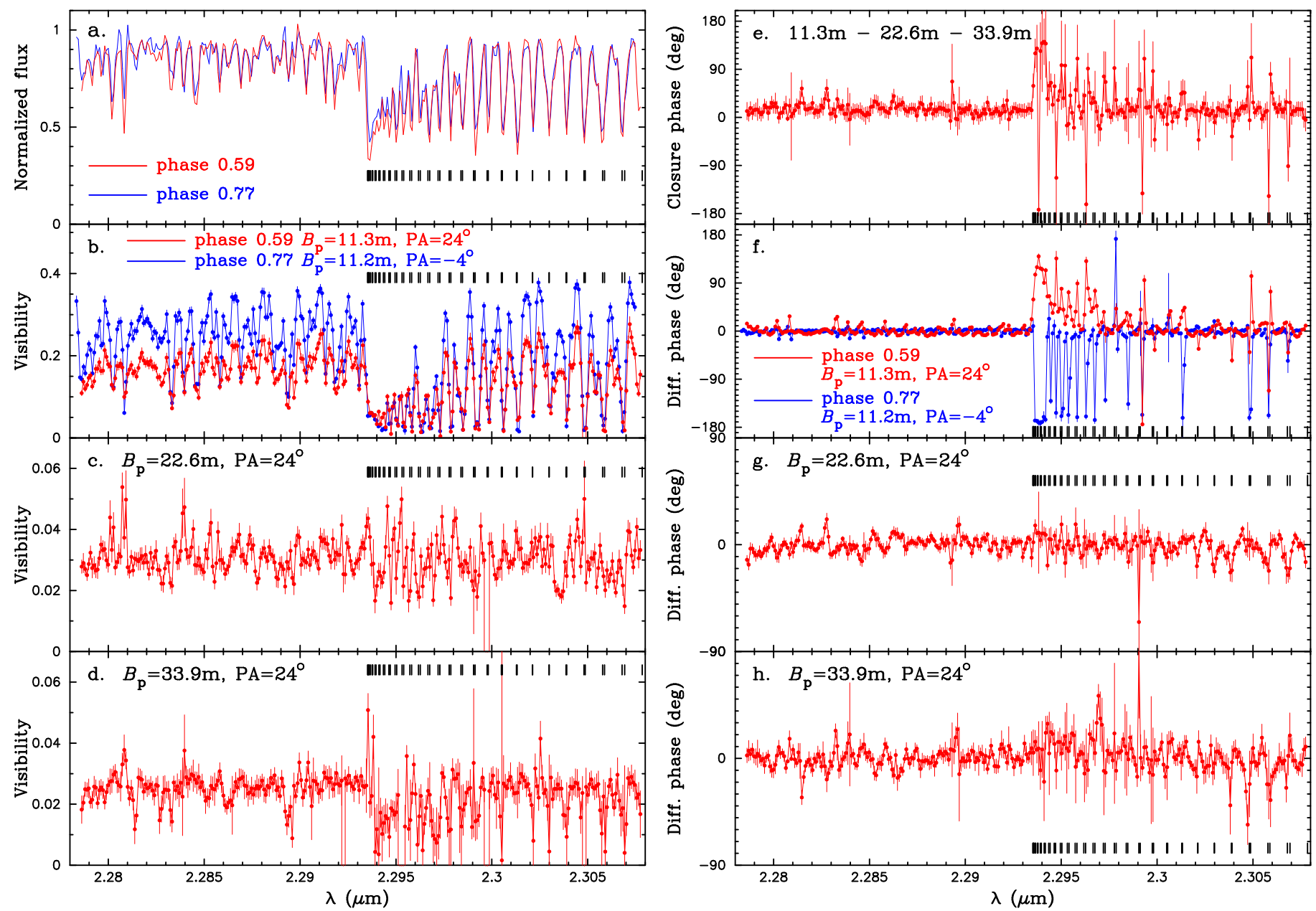

Fig. 6. VLTI/AMBER observations of W Hya with a spectral resolution of 12000 near minimum light (phase 0.59 ) on 2014 February 11 (data set \#3). a) Observed spectrum. b)-d) Visibilities. e) Closure phase. f)-h) Differential phases. The positions of the CO lines are indicated by the ticks. In the panels a), b), and f), the data from Paper I, taken at phase 0.77, are also shown.

directions of the dust clouds. Therefore, the anti-correlation between the inner dust clouds and the $\mathrm{H} \alpha$ emission at large radii, albeit weak, is also consistent with the aforementioned origin of the extended $\mathrm{H} \alpha$ emission.

To study the extended TiO emission in the 2016 data, we first subtracted the flux-calibrated image obtained with the Cnt748 filter from the TiO717 image. However, the resulting image shows negative values in the entire region for the following reason. The TiO band is expected to appear in faint emission (positive pixel values) in the extended atmosphere (i.e., outside the limb of the star), while strong $\mathrm{TiO}$ absorption (negative pixel values) is expected over the stellar disk. When the strong TiO absorption over the stellar disk is convolved with the PSF, it dominates the faint extended emission, resulting in negative values in the entire image. Therefore, we subtracted the TiO717 and Cnt748 images normalized with the intensity at the center of the stellar disk, which was assumed to be at the centroid of the nearly zero-polarization region over the central star (see Fig. 3). This method allows us to recognize the extended emission in positive pixel values and ascertain its spatial extent. On the other hand, because the absolute flux scale is lost, we cannot properly interpret the inhomogeneous absorption over the stellar disk, for example, possible correspondence between the inhomogeneous $\mathrm{TiO}$ absorption seen over the stellar disk and the off-centered bright spot detected in the intensity maps. Figure $5 \mathrm{c}$ shows the continuum-subtracted TiO717 image, which reveals the TiO emission extending to $\sim 150$ mas $\left(\sim 6 R_{\star}\right.$ ), overlapping with the extended $\mathrm{H} \alpha$ emission shown in Fig. 5b.

\subsection{AMBER observations of the central star and molecular outer atmosphere}

Figure 6 shows the visibilities, DPs, and CP measured with VLTI/AMBER. The simultaneously observed spectrum is plotted in Fig. 6a to show the correspondence between the signatures in the spectrum and in the interferometric observables. The spectrum shows the salient $\mathrm{CO}$ first overtone lines longward of the band head at $2.293 \mu \mathrm{m}$. In addition, a number of weak lines due to $\mathrm{H}_{2} \mathrm{O}$ and $\mathrm{CN}$ are present shortward of the band head. Comparison of the spectra taken at two epochs suggests that the $\mathrm{CO}$ band head and the individual lines near the band head $(\$ 2.302 \mu \mathrm{m})$ are slightly deeper at the second epoch (minimum light) than at the first epoch (pre-maximum light). The weak $\mathrm{H}_{2} \mathrm{O}$ and $\mathrm{CN}$ lines shortward of the $\mathrm{CO}$ band head do not indicate significant time variations (the lines at 2.28 and $2.281 \mu \mathrm{m}$ are affected by the residual of the removal of the telluric lines).

The observed visibilities show clear signatures not only in the $\mathrm{CO}$ lines but also in the weak $\mathrm{H}_{2} \mathrm{O}$ and $\mathrm{CN}$ lines. The projected baseline of $11.3 \mathrm{~m}$ of the present data is very close to one of the baselines of the data reported in Paper I (11.2 m), obtained at phase 0.77 . Therefore, the visibility measured at that baseline from Paper I is overplotted in Fig. 6c to show time variations. 
Obviously, the visibility shows more pronounced time variations than the spectra: the visibility obtained near minimum light (phase 0.59 ) is noticeably lower than that obtained at phase 0.77 , suggesting that the object appears larger at minimum light than at a later phase closer to maximum light. This is qualitatively consistent with the time variations in the angular size of W Hya measured from 1.1 to $3.8 \mu \mathrm{m}$ by Woodruff et al. (2009). We note, however, that the position angle of the projected baseline differed by $28^{\circ}$ at two epochs. Therefore, it is possible that deviation from circular symmetry is at least partially responsible for the observed difference in the visibility.

To estimate the angular size of the star, we fit the observed visibilities with a power-law-type limb-darkened disk (Hestroffer et al. 1997). Then we computed the average of the limb-darkened disk diameter and limb-darkening parameter derived at the continuum wavelengths shortward of the band head, avoiding the moderately strong lines. Figure 7 a shows the fit at one of the continuum wavelengths, together with the fit at the same wavelength for the 2015 data. The averaged limb-darkened disk diameter and limb-darkening parameter in the continuum are $49.1 \pm 1.5$ mas and $1.16 \pm 0.49$, respectively. For the data taken at phase 0.77 , we obtained the average limb-darkened disk diameter of $46.6 \pm 0.1$ mas and the limb-darkening parameter of $2.1 \pm 0.2$ (Paper I). Therefore, as can be seen in Fig. 7b, the star appears larger and shows weaker limb-darkening near minimum light than at pre-maximum light. However, Fig. 7a reveals that the data show clear and significant discrepancy from the limbdarkened disk fit. The median of the reduced $\chi^{2}$ at the continuum wavelengths is 29.9 , which also suggest that the star shows significant deviation from a limb-darkened disk.

We note that the AMBER observations in Paper I (2014 April 22) and the present paper (2014 February 11) were carried out in the same variability cycle of W Hya. Therefore, the cycle-to-cycle variation is not responsible for the time variations in the AMBER data. On the other hand, as mentioned in Sect. 2.2, our AMBER observations of $\mathrm{W}$ Hya took place at a phase close to that of the SPHERE/ZIMPOL observations but in a different variability cycle. We estimate, however, that the cycle-dependence of the angular size near $2.3 \mu \mathrm{m}$ is small based on the aperture-masking observations of W Hya over eight variability cycles $(\sim 8.3 \mathrm{yr})$ by Woodruff et al. (2008). The $K$-band $(2.26 \mu \mathrm{m})$ uniform-disk diameters that they derived near minimum light (instead of a limb-darkened disk as we used) range from 41.5 to 45.9 mas. This suggests a cycle-to-cycle variation in the uniform-disk diameter of \pm 2.2 mas. If we adopt this value for the cycle-to-cycle variation in the limb-darkened disk, the estimate of the distance of the clumpy dust clouds in terms of the stellar radius is affected slightly, by $\pm 5 \%$ at most. Therefore, it does not significantly change the interpretation of the results.

For the $\mathrm{CO}$ lines, we could not achieve any reasonable fit to the observed data with a limb-darkened disk. The second and third baselines (projected baseline lengths of 22.6 and $33.9 \mathrm{~m}$ ) correspond to the second and third visibility lobe in the continuum. Since the star appears more extended in the CO lines, these baselines may correspond to even higher visibility lobes in the CO lines. The visibilities at such high lobes are affected by inhomogeneities, whose presence can also be inferred from the non-zero DPs and non-zero/non- $180^{\circ}$ CP (Figs. 6e-h). Probably these inhomogeneities make the fit with a simple, symmetric limb-darkened disk impossible. Nevertheless, to obtain a rough estimate of the spatial extent of the atmosphere in the CO lines, we derived the Gaussian FWHM only from the visibilities at the shortest baseline. The FWHMs in the CO lines range from 40 to 46 mas, and the Gaussian intensity profile in one of the CO lines
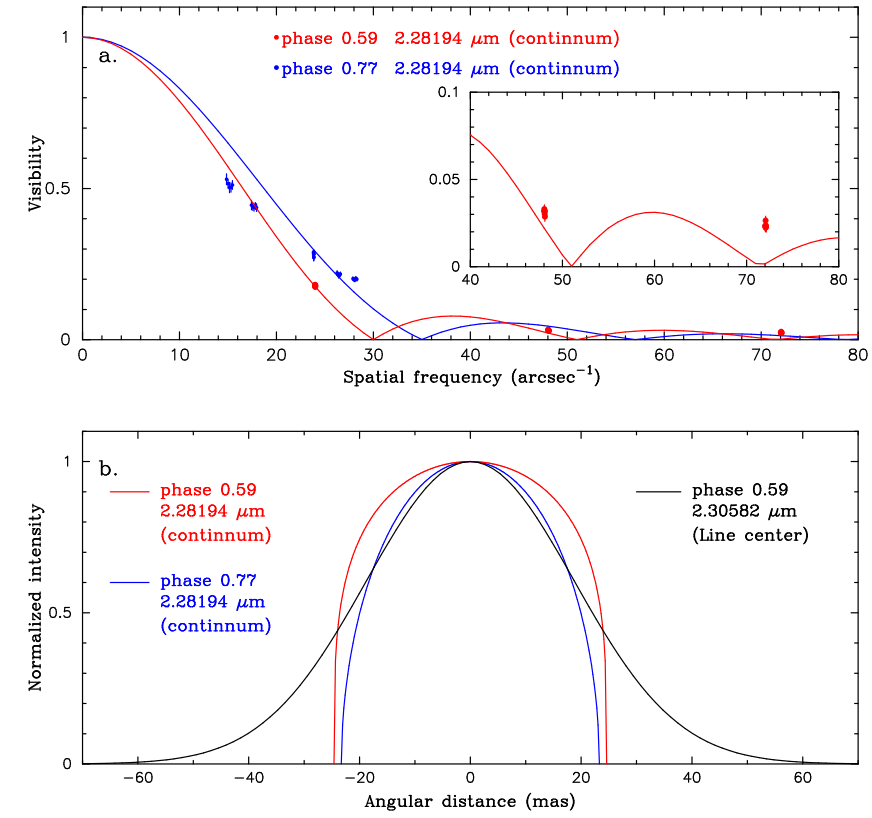

Fig. 7. Limb-darkened disk fit to the AMBER data of W Hya. a) Visibilities observed at a continuum wavelength on 2014 February 11 (phase 0.59) are plotted with the red dots, together with the power-law-type limb-darkened disk fit (red solid line). The inset shows an enlarged view of the second, third, and fourth visibility lobes. The visibilities observed at the corresponding wavelength on 2014 April 22 (phase 0.77) are shown by the blue dots, together with the limb-darkened disk fit (blue solid line). b) Limb-darkened disk intensity profiles derived for phase 0.59 (red solid line) and 0.77 (blue solid line) at the continuum wavelengths shown in the panel a). The Gaussian intensity profile in one of the CO lines obtained at phase 0.59 is plotted by the black solid line.

(at the line center) is plotted in Fig. 7b. The figure suggests that the atmosphere appears more than twice as extended in the $\mathrm{CO}$ lines as in the continuum.

\section{Determination of luminosity and effective temperature at pre-maximum and minimum light}

In Paper I, we adopted an effective temperature of $2500 \mathrm{~K}$ based on the modeling of the spectral energy distribution of W Hya carried out by Khouri et al. (2015) and derived a luminosity of $5130 \mathrm{~K}$ by combining the effective temperature with the angular diameter measured with VLTI/AMBER and the distance of $78 \mathrm{pc}$. However, now that we have SPHERE-ZIMPOL data taken at two distinct phases, it is necessary to use the effective temperature and luminosity appropriate for each epoch.

To derive the bolometric flux at the second epoch (minimum light), we collected (spectro)photometric data as follows. We used the flux derived with five SPHERE-ZIMPOL filters (Table 2) in the visible. For the near-IR domain, we scaled the spectrum observed at phase 0.58 by Woodruff et al. (2009, see their Fig. 4) to an $H$-magnitude of -2.4 , which is a typical value near minimum light based on the long-term photometry by Whitelock et al. (2000). In the wavelength region longward of $2.4 \mu \mathrm{m}$, we adopted the spectrum obtained with the Short Wavelength Spectrometer (SWS) onboard the Infrared Space Telescope (ISO). We downloaded the spectrum observed on 1997 January 7 near minimum light (phase 0.56) from the 
ISO data archive ${ }^{4}$. The collected (spectro)photometric data were dereddened by $A_{V}=0.245$ derived using the parameterization of the interstellar extinction of Arenou et al. (1992). The bolometric flux was derived to be $1.69 \times 10^{-8} \mathrm{~W} \mathrm{~m}^{-2}$. This translates into a bolometric luminosity of $3180_{-440}^{+550} L_{\odot}$ when combined with the distance of $78_{-5.6}^{+6.5} \mathrm{pc}$. The derived bolometric flux and the angular diameter of 49.1 mas measured in the continuum near $2.3 \mu \mathrm{m}$ with VLTI/AMBER lead to an effective temperature of $2140 \mathrm{~K}$.

We also derived the luminosity and the effective temperature at the first-epoch (pre-maximum, phase 0.92) to check whether the values adopted in Paper I can be justified or not. Instead of the flux derived with the SPHERE-ZIMPOL filter, we used the high-resolution visible spectrum presented by Uttenthaler et al. (2011) with our absolute flux calibration described in Paper I. For the near-IR domain, we scaled the spectrum obtained at phase 0.79 by Woodruff et al. (2009, see their Fig. 5) with an $H$-magnitude of -2.6 , which is a value representative of premaximum light based on the aforementioned study of Whitelock et al. (2000). For the wavelength region longward of $2.4 \mu \mathrm{m}$, no ISO/SWS spectrum near pre-maximum is available. Therefore, we scaled the ISO/SWS spectrum taken on 1996 February 14 at phase 0.67 to match the above flux-calibrated spectrum of Woodruff et al. (2009) at $2.4 \mu \mathrm{m}$. The bolometric flux was found to be $2.40 \times 10^{-8} \mathrm{~W} \mathrm{~m}^{-2}$, which translates into a luminosity of $4520_{-630}^{+780} L_{\odot}$ with the distance of $78_{-5.6}^{+6.5} \mathrm{pc}$. The combination of this bolometric flux and the angular diameter of 46.6 mas measured in the continuum in Paper I results in an effective temperature of $2400 \mathrm{~K}$. These luminosity and effective temperature agree reasonably well with the $5130 L_{\odot}$ and $2500 \mathrm{~K}$ adopted in Paper I.

\section{Monte Carlo radiative transfer modeling}

To derive the physical properties of the dust environment close to the star, we used the multi-dimensional dust radiative transfer code mcsim_mpi (Ohnaka et al. 2006), adopting the shell with a cone-shaped density enhancement used in Paper I. The radiation from the central star was approximated with the blackbody with $2140 \mathrm{~K}$ as derived in the previous section. The dust shell is defined by its inner and outer boundary radii. The inner boundary radius is defined so that the dust temperature reaches a sublimation temperature of $1500 \mathrm{~K}$. The dust density was assumed to be $\propto r^{-p}$, and $p$ was fixed to 3 in Paper I, which approximately represents the density distribution in the wind acceleration region (see discussion in Sect. 4 of Paper I). However, as Fig. 4 shows, the dust distribution is more extended in 2016 than observed in 2015, which suggests that the optical depth is higher and/or the density gradient is shallower in 2016 than derived from the 2015 data. Therefore, in the present work, we treated $p$ as a free parameter.

The clumpy dust clouds seen in the SPHERE-ZIMPOL data are approximated with a cone-shaped density enhancement in a certain direction (see Fig. 8a of Paper I). We adopted the halfopening angle of the density enhancement of $45^{\circ}$ and a density ratio of 4 as derived in Paper I, because the overall structure of the inner dust clouds seen in 2016 is similar to what was seen in 2015. For the dust opacities, we computed the absorption and scattering coefficients as well as the scattering matrix elements for spherical grains using the code of Bohren \& Huffman (1983) from the complex refractive indices of $\mathrm{Al}_{2} \mathrm{O}_{3}$ measured by Koike et al. (1995, their "Alumina" sample), $\mathrm{MgSiO}_{3}$, and $\mathrm{Mg}_{2} \mathrm{SiO}_{4}$ measured by Jäger et al. (2003). The free parameters of our modeling are the optical depth of the dust shell in the radial direction,

\footnotetext{
4 http://www. cosmos.esa.int/web/iso/ access-the-archive
}

the outer radius of the dust shell, the exponent of the density distribution $p$, and the grain size. We compared the model images convolved with the observed PSFs with the maps of the intensity and the degree of polarization observed at 645,748 , and $820 \mathrm{~nm}$, as well as the polarized intensity normalized with its peak as a byproduct. We avoided $656.3 \mathrm{~nm}$ and $717 \mathrm{~nm}$, because the intensity maps at these wavelengths are affected by the extended $\mathrm{H} \alpha$ and $\mathrm{TiO}$ emission, respectively, as described in Sect. 3.1.2.

Figure 8 shows the best-fit model, which is characterized by a $550 \mathrm{~nm}$ optical depth of 0.6 , an inner radius of $1.3 R_{\star}$, an outer radius of $10 R_{\star}$, a density distribution exponent of 3 , and $\mathrm{Al}_{2} \mathrm{O}_{3}$ grains with a size of $0.1 \mu \mathrm{m}$. The dust mass of this model is $3.7 \times 10^{-9} M_{\odot}$ with a bulk density of $4 \mathrm{~g} \mathrm{~cm}^{-3}$ adopted for $\mathrm{Al}_{2} \mathrm{O}_{3}$. The uncertainty in the $550 \mathrm{~nm}$ optical depth, outer radius, and density distribution exponent is $\pm 0.2, \pm 2$, and \pm 0.5 , respectively.

Figure 9 shows the 1D profiles of the observed and model images at eight different position angles $\left(0^{\circ}, 45^{\circ}, 90^{\circ}, \ldots, 315^{\circ}\right)$. To avoid the bright, off-centered spot in the observed intensity maps, the center of the images was assumed to be the centroid of the nearly zero-polarization region over the central star. Figure 9 (top row) shows that the intensity maps predicted by the model are narrower than the observed data, particularly at 645 and $748 \mathrm{~nm}$. The bright, off-centered spot seen in the observed intensity maps makes the 1D cuts broader, which could partially explain the discrepancy between the predicted intensity maps and the observed data. However, the discrepancy at 645 and $748 \mathrm{~nm}$ seems to be difficult to explain by this effect alone. The discrepancy may primarily be attributed to the extended TiO emission, as presented in Fig. 5c. The wavelengths sampled with the CntHa, Cnt748, and Cnt820 filters are not entirely free of the effects of the $\mathrm{TiO}$ bands, because the $\mathrm{TiO}$ bands are dominant in the visible. Therefore, the extended $\mathrm{TiO}$ emission may be present at 645 and $748 \mathrm{~nm}$, which can make the intensity 1D cuts broader. On the other hand, the observed normalized polarized intensity and the degree of polarization are reproduced reasonably well. The shape of the polarized intensity profiles and the absolute values of the degree of polarization agree with the observed data. Moreover, given the simplicity of the model, the observed position angle dependence of the polarized intensity and the degree of polarization is also reproduced well.

We found out that the models with grain sizes larger than $0.2 \mu \mathrm{m}$ predict the degree of polarization at $645 \mathrm{~nm}$ to be too low compared to the observations. The models with grains smaller than $0.05 \mu \mathrm{m}$ also predict the degree of polarization to be too low. In these models, the amount of scattered light is much smaller, because the albedos are much lower for grains smaller than $0.05 \mu \mathrm{m}$. The decrease in scattered light means a larger fraction of the unpolarized direct star light. Therefore, after convolving with the PSFs, the degree of polarization becomes lower. As in the case of Paper I, the models with $\mathrm{Mg}_{2} \mathrm{SiO}_{4}$ and $\mathrm{MgSiO}_{3}$ also reproduce the observed data, and therefore, we cannot constrain the grain species from our SPHERE-ZIMPOL data alone.

In Paper I, we also used the fraction of scattered light derived from the near-IR polarimetric interferometric observations of Norris et al. (2012) as observational constraints. The fraction of scattered light computed from the model shown in Figs. 8 and 9 is $0.06,0.03$, and 0.004 at $1.04,1.24$, and $2.06 \mu \mathrm{m}$, respectively, which is much lower than the observed values of $0.176 \pm 0.002$ $(1.04 \mu \mathrm{m}), 0.110 \pm 0.003(1.24 \mu \mathrm{m})$, and $0.022 \pm 0.004(2.06 \mu \mathrm{m})$. However, the observations of Norris et al. (2012) took place at phase 0.2 , very different from the 0.54 of our second-epoch SPHERE-ZIMPOL observations. Given the noticeable time variations seen in the observed maps of the polarized intensity and degree of polarization between phase 0.92 and 0.54 (Figs. 2-4), 


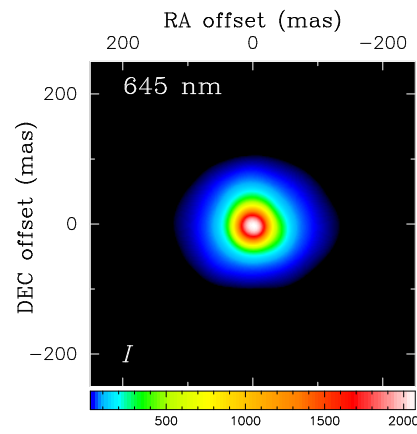

RA offset (mas)
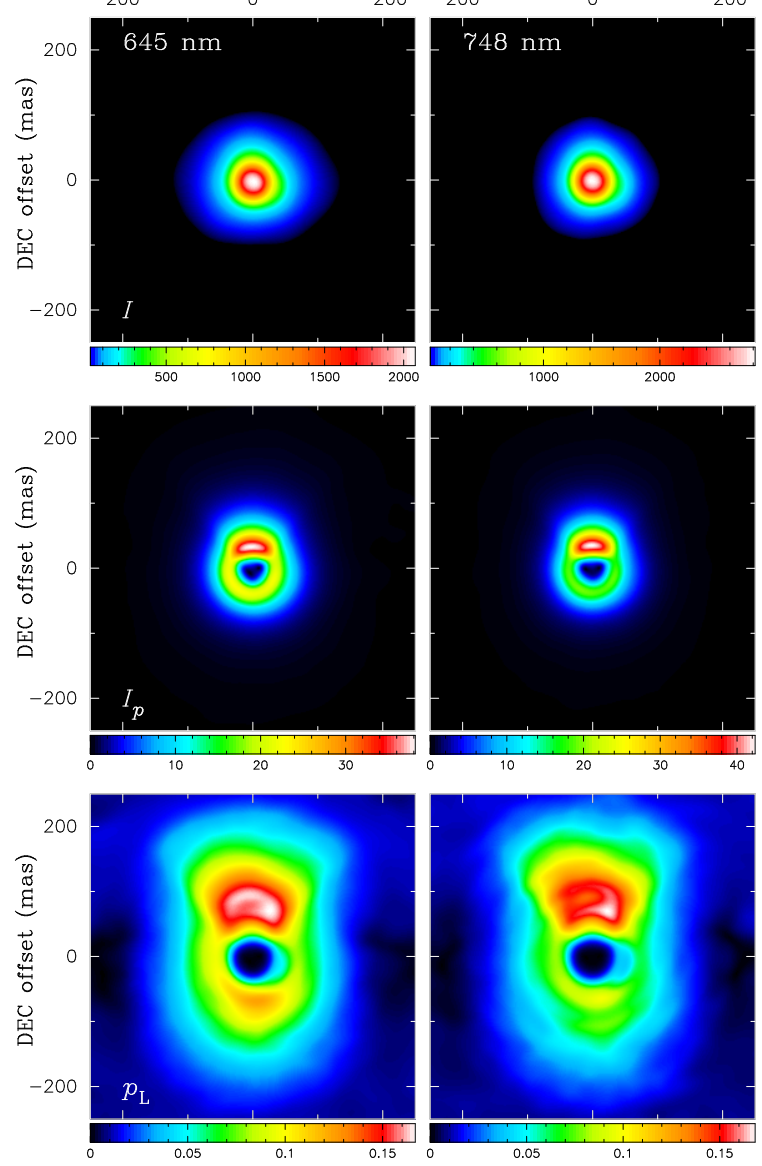

RA offset (mas)
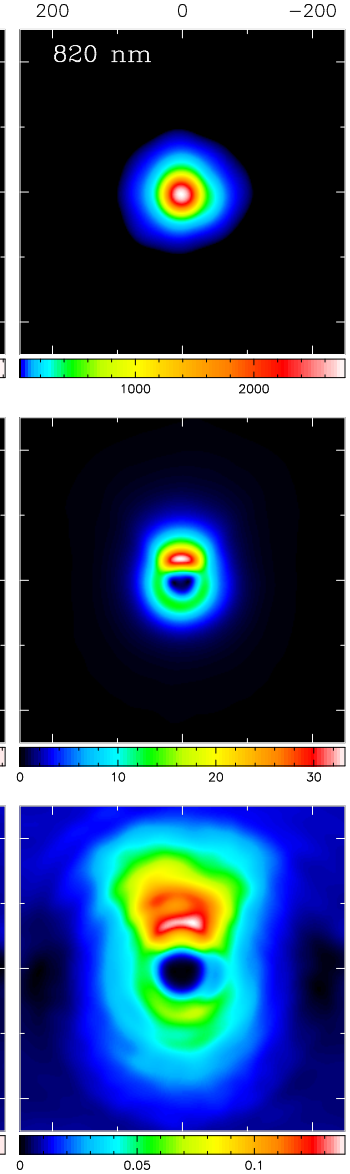

Fig. 8. Dust clump model for W Hya. The top, middle, and bottom rows show the maps of the intensity, polarized intensity, and degree of linear polarization predicted by the model with the convolution with the observed PSF. The model images predicted at 645, 748, and $820 \mathrm{~nm}$ are shown in the first, second, and third columns. North is up, east to the left. it would not be surprising that the fraction of scattered light changes significantly between phase 0.2 and 0.54 . Therefore, we did not attempt to reproduce the results of Norris et al. (2012) in the present modeling.

Our modeling of the 2015 data presented in Paper I suggested the predominance of grains as large as $0.5 \mu \mathrm{m}$. To check whether or not the presence of such large grains can be excluded for the 2016 data, we computed models with $0.1 \mu \mathrm{m}$ and $0.5 \mu \mathrm{m} \mathrm{Al}_{2} \mathrm{O}_{3}$ grains. For simplicity, we set the inner and outer radii of the distribution of two grain species as well as the exponent of the density distribution to be the same as those of the best-fit model with $0.1 \mu \mathrm{m} \mathrm{Al}{ }_{2} \mathrm{O}_{3}$ grains alone. It turned out that the models with $\tau_{500 \mathrm{~nm}}=0.8(0.1 \mu \mathrm{m}$ grains $)$ and $\tau_{500 \mathrm{~nm}} \leq 0.1(0.5 \mu \mathrm{m}$ grains) can fairly reproduce the SPHERE-ZIMPOL data. These optical depths correspond to dust mass of $4.9 \times 10^{-9} M_{\odot}$ and $\leq 5.5 \times 10^{-10} M_{\odot}$ for $0.1 \mu \mathrm{m}$ and $0.5 \mu \mathrm{m}$ grains, respectively. The $550 \mathrm{~nm}$ optical depth of the $0.5 \mu \mathrm{m}$ grains in the best-fit model for the 2015 data is 0.1 . Therefore, we cannot exclude the presence of $0.5 \mu \mathrm{m}$ grains with an amount comparable to what was found in 2015 .

\section{Discussion}

Our modeling of the second-epoch SPHERE-ZIMPOL data suggests the predominance of $0.1 \mu \mathrm{m}$ grains, noticeably smaller than the $0.5 \mu \mathrm{m}$ found for the first-epoch data. One may wonder if the presence of small, $0.1 \mu \mathrm{m}$ grains was not found by the modeling in Paper I, simply because we assumed a single grain size. Therefore, we computed models with $0.1 \mu \mathrm{m}$ and $0.5 \mu \mathrm{m} \mathrm{Al} \mathrm{Al}_{2} \mathrm{O}_{3}$ grains and compared with the 2015 data in the same manner as described in Paper I. We compared the models with the $I_{\mathrm{P}}$ maps at 645,717 , and $820 \mathrm{~nm}$ normalized with its peak value and the fraction of scattered light in the near-IR derived by Norris et al. (2012). In the modeling of the 2015 data, the $I$ and $p_{\mathrm{L}}$ maps were not used because the PSF references taken with Strehl ratios much lower than those for W Hya made it impossible to convolve the model images with PSFs appropriate for comparison with the observed data. The parameters of the central star were set to be identical with those adopted in Paper I. We set the inner and outer boundary radii as well as the density distribution exponent to be the same for $0.1 \mu \mathrm{m}$ and $0.5 \mu \mathrm{m}$ grains, adopting the values derived in Paper I: the inner and outer radius of 1.9 and $3.0 R_{\star}$, respectively, and the density distribution exponent of 3 . We found out that the models with $\tau_{500 \mathrm{~nm}} \leq 0.2(0.1 \mu \mathrm{m}$ grains $)$ and $\tau_{500 \mathrm{~nm}}=0.1(0.5 \mu \mathrm{m}$ grains $)$ can reproduce the $I_{\mathrm{P}}$ maps obtained at the first epoch and the fraction of scattered light in the near-IR. The corresponding dust mass is $8.8 \times 10^{-10} M_{\odot}$ and $3.9 \times 10^{-10} M_{\odot}$ for the 0.1 and $0.5 \mu \mathrm{m}$ grains, respectively. Therefore, it is possible that grains as small as $0.1 \mu \mathrm{m}$ were present in 2015.

The parameters of the $0.1 \mu \mathrm{m}+0.5 \mu \mathrm{m}$ grain models derived for the 2015 and 2016 observations are listed in Table 4. It should be noted that the fraction of the large $0.5 \mu \mathrm{m}$ grains is different at the two epochs. The ratio of the mass of the $0.5 \mu \mathrm{m}$ grains to that of the $0.1 \mu \mathrm{m}$ grains $\left(M_{\text {dust }}^{0.5 \mu \mathrm{m}} / M_{\text {dust }}^{0.1 \mu \mathrm{m}}\right)$ is higher than 0.42 at pre-maximum light in 2015 , while it is lower than 0.11 at minimum light in 2016. Therefore, our modeling at two epochs suggests the predominance of grains as large as $0.5 \mu \mathrm{m}$ at premaximum light and the predominance of $0.1 \mu \mathrm{m}$ grains at minimum light.

Gobrecht et al. (2016) present non-equilibrium gas-dust chemistry models for the Mira star IK Tau. Given its larger variability amplitude and its higher mass-loss rate than that of 


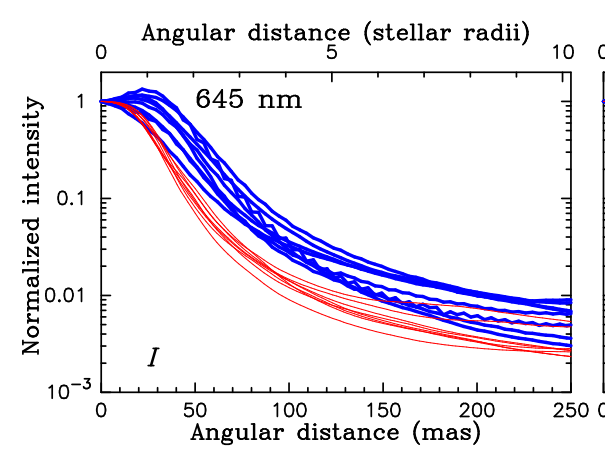

Angular distance (stellar radii)
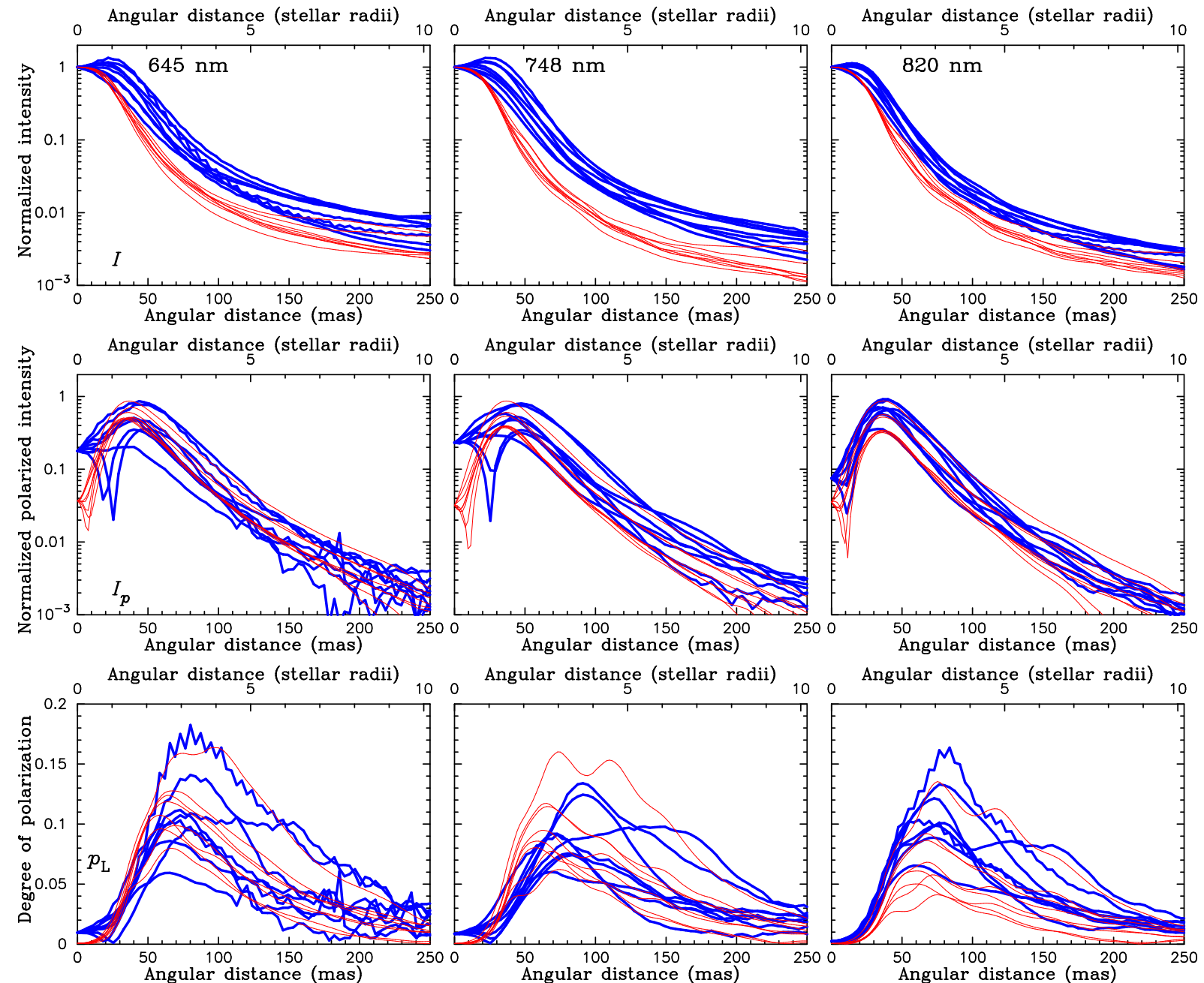

(mas)

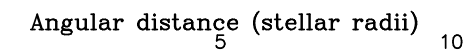

Fig. 9. Comparison of the dust clump model and the observed SPHERE-ZIMPOL data. The top, middle, and bottom rows show the 1D cuts at eight different position angles $\left(0^{\circ}, 45^{\circ}, 90^{\circ}, \ldots, 315^{\circ}\right)$ of the intensity, normalized polarized intensity, and degree of polarization maps, respectively. The first, second, and third columns show the comparison at 645,748 , and $820 \mathrm{~nm}$, respectively. In each panel, the observed data are plotted with the thick blue lines, while the model is plotted with the thin red lines.

W Hya, IK Tau is considered to be more evolved than W Hya. Their models do not include the dynamical effects caused by the radiation pressure on dust grains. Still, it may be worth comparing the results of such theoretical models with the phase dependence of the grain size suggested by our modeling. Their models show that the passage of shocks destroys grains completely, and small grains start to form only half a period after the passage of the shocks, followed by efficient grain growth. The change in the characteristic grain size between the two epochs can be interpreted based on this theoretical prediction. Possibly, the first epoch at pre-maximum light in 2015 may correspond to the phase when the efficient grain growth was occurring, while the second epoch at minimum light may correspond to the phase when small grains just started to form. Their models also show that silicate dust forms at radii larger than $3.5 R_{\star}$, while the formation of $\mathrm{Al}_{2} \mathrm{O}_{3}$ dust is restricted at radii smaller than $2 R_{\star}$. It is possible that the polarization detected over the more extended region in 2016 may be attributed to the formation of silicate.

In contrast to the models of Gobrecht et al. (2016), Höfner et al. (2016) present dynamical models in which the grain growth and the radiation pressure on dust grains are taken into account in a self-consistent manner, but the amount of the seed nuclei for dust formation is treated as a free parameter. They also included the formation of silicate mantle on $\mathrm{Al}_{2} \mathrm{O}_{3}$ grains, which was previously proposed by, e.g., Kozasa \& Sogawa (1997a,b). The models show the pulsation phase-dependence of the grain size and the formation of silicate mantle on $\mathrm{Al}_{2} \mathrm{O}_{3}$ core farther out than $\mathrm{Al}_{2} \mathrm{O}_{3}$ grains (see their Fig. 9). Therefore, the formation of grains in the extended region found in 2016 may indicate the formation of silicate mantle on $\mathrm{Al}_{2} \mathrm{O}_{3}$ grains, instead of pure silicate as modeled by Gobrecht et al. (2016). However, given that we cannot differentiate between the models with $\mathrm{Al}_{2} \mathrm{O}_{3}$, $\mathrm{Mg}_{2} \mathrm{SiO}_{4}$, and $\mathrm{MgSiO}_{3}$ as mentioned in Sect. 5, it is not possible to draw a definitive conclusion about the nature of the dust found in the extended region. The models of Höfner et al. (2016) also suggest that the radiation pressure on $\mathrm{Al}_{2} \mathrm{O}_{3}$ grains forming very close to the star is too low to drive mass loss, and $\mathrm{Al}_{2} \mathrm{O}_{3}$ grains with silicate mantle forming farther out may be more efficient in driving stellar winds by the scattering of stellar photons. SPHERE-ZIMPOL observations with higher cadence will help us to better understand the dynamics of the dust clouds and clarify where the effective wind acceleration takes place.

\section{Concluding remarks}

We have presented second-epoch visible polarimetric imaging observations of the AGB star W Hya with VLT/SPHEREZIMPOL at minimum light (phase 0.54), which is distinct from 
Table 4. Summary of the parameters derived from the modeling with $0.1 \mu \mathrm{m}$ and $0.5 \mu \mathrm{m}$ grains for the observations at two epochs.

\begin{tabular}{lcc}
\hline \hline Parameter & $\begin{array}{c}\text { 2015 July 08 } \\
\text { phase 0.92 }\end{array}$ & $\begin{array}{c}\text { 2016 March 23 } \\
\text { phase 0.54 }\end{array}$ \\
\hline$T_{\text {eff }}(\mathrm{K})$ & 2500 & 2140 \\
$L_{\star}\left(L_{\odot}\right)$ & 5130 & 3200 \\
Inner radius & 1.9 & 1.3 \\
Outer radius & 3.0 & 10.0 \\
Density exponent & 3 & 3 \\
\hline$\tau_{500 \mathrm{~nm}}(0.1 \mu \mathrm{m})$ & $\leq 0.2$ & 0.8 \\
$M_{\mathrm{dust}}^{0.1 \mu \mathrm{m}}\left(M_{\odot}\right)$ & $\leq 8.8 \times 10^{-10}$ & $4.9 \times 10^{-9}$ \\
\hline$\tau_{500 \mathrm{~nm}}(0.5 \mu \mathrm{m})$ & 0.1 & $\leq 0.1$ \\
$M_{\mathrm{dust}}^{0.5 \mu \mathrm{m}}\left(M_{\odot}\right)$ & $3.9 \times 10^{-10}$ & $\leq 5.5 \times 10^{-10}$ \\
\hline$M_{\mathrm{dust}}^{0.5 \mu \mathrm{m}} / M_{\mathrm{dust}}^{0.1 \mu \mathrm{m}}$ & $\geq 0.42$ & $\leq 0.11$ \\
\hline
\end{tabular}

the first-epoch observations at pre-maximum light (phase 0.92). We have also reported on high-spectral resolution long-baseline interferometric observations of W Hya near $2.3 \mu \mathrm{m}$ with VLTI/AMBER near minimum light.

The polarized intensity maps obtained at five wavelengths from 645 to $820 \mathrm{~nm}$ with SPHERE-ZIMPOL reveal three clumpy dust clouds at 34-50 mas (1.4-2 $\left.R_{\star}\right)$. Comparison of the data obtained at two epochs (phase 0.92 and 0.54) shows clear time variations in the clumpy dust clouds close to the star. The degree of linear polarization measured at minimum light is systematically higher than that measured at pre-maximum light, and the polarization signals were detected in a noticeably more extended region compared to the first epoch. The continuumsubtracted $\mathrm{H} \alpha$ emission observed at minimum light extends to $\sim 150$ mas, also exhibiting time variations between premaximum light and minimum light. The continuum-subtracted TiO image obtained at minimum light reveals $\mathrm{TiO}$ emission extending to $\sim 150$ mas $\left(\sim 6 R_{\star}\right)$, overlapping with the $\mathrm{H} \alpha$ emission.

Our VLTI/AMBER data have also revealed time variations in the extended atmosphere. The fitting to the visibilities at the continuum wavelengths near $2.3 \mu \mathrm{m}$ with a power-law-type limbdarkened disk has resulted in a limb-darkened disk diameter of $49.1 \pm 1.5$ mas and a limb-darkening parameter of $1.16 \pm 0.49$, which means that the atmosphere is more extended with weaker limb-darkening compared to pre-maximum light.

Our 2D Monte Carlo radiative transfer modeling shows that the SPHERE-ZIMPOL data obtained at minimum light can be explained by a shell with $\sim 0.1 \mu \mathrm{m}$ grains of $\mathrm{Al}_{2} \mathrm{O}_{3}$ or $\mathrm{Mg}_{2} \mathrm{SiO}_{4}$ or $\mathrm{MgSiO}_{3}$ with a $550 \mathrm{~nm}$ optical depth of $0.6 \pm 0.2$ and an inner and outer radius of $1.3 R_{\star}$ and $10 \pm 2 R_{\star}$, respectively. Our modeling suggests the predominance of $0.1 \mu \mathrm{m}$ grains, in marked contrast to the predominance of large $(\sim 0.5 \mu \mathrm{m})$ grains at premaximum light. This implies that small grains might just have started to form at minimum light, while the efficient grain growth might already have been taking place at pre-maximum light.

High-cadence monitoring of the time evolution of the clumpy dust clouds as well as the extended $\mathrm{H} \alpha$ and $\mathrm{TiO}$ emission is of great important for understanding the interplay between the pulsation-induced shocks and the molecule and dust formation. Given that the morphology of the images of R Dor changed within 48 days (Khouri et al. 2016), SPHERE-ZIMPOL observations of W Hya as frequent as every few weeks are useful for clarifying the physical process behind the time variations. Milliarcsecond-resolution velocity-resolved aperture-synthesis imaging of the extended atmosphere with VLTI/AMBER or VLTI/GRAVITY (Eisenhauer et al. 2008) is crucial for examining whether or not the gas at the locations of the dust clouds shows systematic outward motions. Moreover, mid-IR spectro-interferometric imaging with VLTI/MATISSE (Lopez et al. 2014) will allow us to extract spatially resolved dust spectra, which will be indispensable for better understanding dust chemistry within $\sim 10 R_{\star}$.

Acknowledgements. We thank the ESO Paranal team for supporting our SPHERE and AMBER observations. We are also grateful to the referee Susanne Höfner for her valuable comments, particularly on the interpretation of the extended $\mathrm{H} \alpha$ emission. This research made use of the SIMBAD database, operated at the CDS, Strasbourg, France. We acknowledge with thanks the variable star observations from the AAVSO International Database contributed by observers worldwide and used in this research. K.O. acknowledges a grant from Universidad Católical del Norte.

\section{References}

Arenou, F., Grenon, M., \& Gómez, A. 1992, A\&A, 258, 104

Beuzit, J.-L., Feldt, M., Dohlen, K., et al. 2008, SPIE Proc., 7014, 18

Bohren, C. F., \& Huffman, D. R. 1983, Absorption and Scattering of Light by Small Particles (New York: Wiley)

Cardelli, J. A., Clayton, G. C., \& Mathis, J. S. 1989, ApJ, 345, 245

Chelli, A., Hernandez Utrera, O., \& Duvert, G. 2009, A\&A, 502, 705

Eisenhauer, F., Perrin, G., Brandner, W., et al. 2008, SPIE Proc., 7013, 70132A

Fleischer, A. J., Gauger, A., \& Sedlmayr, E. 1991, A\&A, 242, L1

Fleischer, A. J., Gauger, A., \& Sedlmayr, E. 1992, A\&A, 266, 321

Fox, M. W., Wood, P. R., \& Dopita, M. A. 1984, A\&A, 286, 337

Gail, H.-P., Scholz, M., \& Pucci, A. 2016, A\&A, 591, A17

Gobrecht, D., Cherchneff, I., Sarangi, A., Plane, J. M. C., \& Bromley, S. T. 2016, A\&A, 585, A6

Hestroffer, D. 1997, A\&A, 327, 199

Höfner, S. 2008, A\&A, 491, L1

Höfner, S., Bladh, S., Aringer, B., \& Ajuja, R. 2016, A\&A, 594, A108

Ireland, M. J., Tuthill, P. G., Bedding, T. R., Robertson, J. G., \& Jacob, A. P. 2004, MNRAS, 350, 365

Jäger, C., Dorschner, J., Mutschke, H., Posch, T., \& Henning, Th. 2003, A\&A, 408, 193

Jeong, K. S., Winters, J. M., Le Bertre, T., \& Sedlmayr, E. 2003, A\&A, 407, 191 Kervella, P., Thévenin, F., Ségransan, D., et al. 2003, A\&A, 404, 1087

Khouri, T., Waters, L. B. F. M., de Koter, A., et al. 2015, A\&A, 577, A114

Khouri, T., Maercker, M., Waters, L. B. F. M., et al. 2016, A\&A, 591, A70

Knapp, G. R., Pourbaix, D., Platais, I., \& Jorissen, A. 2003, A\&A, 403, 993

Koike, C., Kaito, C., Yamamoto, T., et al. 1995, Icarus, 114, 203

Kozasa, T., \& Sogawa, H. 1997a, Ap\&SS, 251, 165

Kozasa, T., \& Sogawa, H. 1997b, Ap\&SS, 255, 437

Lopez, B., Lagarde, S., Jaffe, W., et al. 2014, The Messenger, 157, 5

McDonald, I., Zijlstra, A. A., \& Boyer, M. L. 2012, MNRAS, 427, 343

Norris, B., Tuthill, P. G., Ireland, M. J., et al. 2012, Nature, 484, 220

Ohnaka, K., Driebe, T., Hofmann, K.-H., et al. 2006, A\&A, 445, 1015

Ohnaka, K., Hofmann, K.-H., Benisty, M., et al. 2009, A\&A, 503, 183

Ohnaka, K., Weigelt, G., Millour, F., et al. 2011, A\&A, 529, A163

Ohnaka, K., Hofmann, K-.H., Schertl, D., et al. 2013, A\&A, 555, A24

Ohnaka, K., Weigelt, G., \& Hofmann, K.-H. 2016, A\&A, 589, A91 (Paper I)

Petrov, R. G., Malbet, F., Weigelt, G., et al. 2007, A\&A, 464, 1

Pickles, A. J. 1998, PASJ, 110, 863

Tatulli, E., Millour, F., Chelli, A., et al. 2007, A\&A, 464, 29

Thalmann, C., Schmid, H. M., Boccaletti, A., et al. 2008, SPIE Proc., 70143F, 12

Uttenthaler, S., Van Stiphout, K., Voet, K., et al. 2011, A\&A, 531, A88

Whitelock, P., Marang, F., \& Feast, M. 2000, MNRAS, 319, 728

Woodruff, H. C., Tuthill, P. G., Monnier, J. D., et al. 2008, ApJ, 673, 418

Woodruff, H. C., Ireland, M. J., Tuthill, P. G., et al. 2009, ApJ, 691, 1328 